\title{
DIFFERENTIAL EQUATIONS COMPATIBLE WITH BOUNDARY RATIONAL QKZ EQUATION
}

\author{
YOSHIHIRO TAKEYAMA
}

Dedicated to Professor Tetsuji Miwa on his sixtieth birthday

\begin{abstract}
We give differential equations compatible with the rational qKZ equation with boundary reflection. The total system contains the trigonometric degeneration of the bispectral qKZ equation of type $\left(C_{n}^{\vee}, C_{n}\right)$ which in the case of type $G L_{n}$ was studied by van Meer and Stokman. We construct an integral formula for solutions to our compatible system in a special case.
\end{abstract}

\section{INTRODUCTION}

In this paper we give differential equations compatible with the rational version of the quantum Knizhnik-Zamolodchikov (qKZ) equation 8] with boundary reflection, which we call the boundary rational $q K Z$ equation.

Let $V=\mathbb{C}^{2 N}$ be a vector space of even dimension. The boundary rational qKZ equation is the following system of difference equations for an unknown function $f(x \mid y)$ on $\left(\mathbb{C}^{\times}\right)^{N} \times \mathbb{C}^{n}$ taking values in $V^{\otimes n}$ :

$$
\begin{aligned}
& f\left(x \mid \ldots, y_{m}-c, \ldots\right) \\
& =R_{m, m-1}\left(y_{m}-y_{m-1}-c\right) \cdots R_{m, 1}\left(y_{m}-y_{1}-c\right) K_{m}\left(y_{m}-c / 2 \mid x, \beta\right) \\
& \times R_{1, m}\left(y_{1}+y_{m}\right) \cdots R_{m-1, m}\left(y_{m-1}+y_{m}\right) R_{m, m+1}\left(y_{m}+y_{m+1}\right) \cdots R_{m, n}\left(y_{m}+y_{n}\right) \\
& \times K_{m}\left(y_{m} \mid \underline{1}, \alpha\right) R_{m, n}\left(y_{m}-y_{n}\right) \cdots R_{m, m+1}\left(y_{m}-y_{m+1}\right) f\left(x \mid \ldots, y_{m}, \ldots\right)
\end{aligned}
$$

for $1 \leq m \leq n$, where $c, \alpha$ and $\beta$ are parameters and $1=(1, \ldots, 1)$. The linear operator $R(\lambda)$ on $V^{\otimes 2}$ is the rational $R$-matrix, and $K(\lambda \mid x, \beta) \in \operatorname{End}(V)$ is the boundary $K$-matrix which is a linear sum of the identity and the reflection of the basis of $V$ with exponent $x=\left(x_{1}, \ldots, x_{N}\right)$ (see (2.2) below). The indices of $R$ and $K$ in the right hand side signify the position of the components of $V^{\otimes n}$ on which they act. The boundary rational qKZ equation can be regarded as a slight generalization of an additive degeneration of Cherednik's trigonometric qKZ equation [2] associated with the root system of type $C_{n}$. Such equation was also derived as that for correlation functions of the integrable spin chains with a boundary [10, 3]. Recently combinatorial aspects of a special polynomial solution have attracted attention [1, 5, 9]. 
In this paper we give commuting differential operators in the form

$$
D_{a}(x \mid y)=c x_{a} \frac{\partial}{\partial x_{a}}+L_{a}(x \mid y) \quad(1 \leq a \leq N)
$$

where $L_{a}(x \mid y)(1 \leq a \leq N)$ are commuting linear operators acting on $V^{\otimes n}$, and prove that the system consisting of the boundary rational qKZ equation and the differential equations $D_{a}(x \mid y) f(x \mid y)=0(1 \leq a \leq N)$ is compatible (see Theorem 3.3 below). Such compatible system is obtained for the differential KZ or the qKZ equations without boundary reflection by Etingof, Felder, Markov, Tarasov and Varchenko in more general settings [6, 17, 15, 16].

In [11] van Meer and Stokman constructed a consistent system of $q$-difference equations, which they call the bispectral qKZ equation, using the double affine Hecke algebra (DAHA) [4] of type $G L_{n}$. The key ingredients are Cherednik's intertwiners and the dual anti-involution. As mentioned in [11, their construction can be extended to arbitrary root system. In this paper we consider the case of type $\left(C_{n}^{\vee}, C_{n}\right)$. The DAHA of this type also has intertwiners and dual anti-involution [14], and hence we can construct the bispectral qKZ equation. Now recall that the DAHA has a trigonometric degeneration [4]. In this degeneration the bispectral qKZ equation turns into a system of differential equations and additive difference equations. We prove that the system is contained in our compatible system of differential equations and the boundary rational qKZ equation with $N=n$.

As previously mentioned the boundary rational qKZ equation can be regarded as an additive degeneration of the qKZ equation on the root system of type $C_{n}$. For the qKZ equation of type $C_{n}$, Mimachi obtained an integral formula for solutions in a special case [12. Similar construction works for the boundary rational qKZ equation with $\alpha=\beta=k / 2$, where $k$ is a parameter contained in the $R$-matrix, and the exponent $x$ restricted to the hyperplane $x_{2}=\cdots=x_{N}=1$. We prove that the solutions obtained in this way satisfy the differential equation $D_{1}\left(x_{1}, 1, \ldots, 1 \mid y\right) f\left(x_{1} \mid y\right)=0$. Thus we get solutions to our compatible system in a special case.

The rest of this paper is organized as follows. In Section 2 we give the definition of the boundary rational qKZ equation. In Section 3 we construct the commuting differential operators and prove that they are compatible with the boundary rational qKZ equation. In Section 4 we calculate the trigonometric degeneration of the bispectral qKZ equation of type $\left(C_{n}^{\vee}, C_{n}\right)$ and see that it is contained in our compatible system. In Section 5 we give an integral formula for solutions to our compatible system with $\alpha=\beta=k / 2$ and $x=\left(x_{1}, 1, \ldots, 1\right)$.

\section{BOUNDARY RATIONAL QKZ EQUATION}

Let $n$ and $N$ be positive integers, and $c, k, \alpha$ and $\beta$ be generic non-zero complex numbers. Let $V=\oplus_{a=1}^{N}\left(\mathbb{C} v_{a} \oplus \mathbb{C} v_{\bar{a}}\right)$ be a vector space with the basis $\left\{v_{l}\right\}_{l=1, \ldots, N, \overline{1}, \ldots, \bar{N}}$. 
The rational $R$-matrix acting on $V^{\otimes 2}$ is defined by

$$
R(\lambda):=\frac{\lambda+k P}{\lambda+k}
$$

where $P$ is the transposition $P(u \otimes v):=v \otimes u$. It is a rational solution of the Yang-Baxter equation

$$
\begin{aligned}
& R_{12}\left(\lambda_{1}-\lambda_{2}\right) R_{13}\left(\lambda_{1}-\lambda_{3}\right) R_{23}\left(\lambda_{2}-\lambda_{3}\right) \\
& \quad=R_{23}\left(\lambda_{2}-\lambda_{3}\right) R_{13}\left(\lambda_{1}-\lambda_{3}\right) R_{12}\left(\lambda_{1}-\lambda_{2}\right)
\end{aligned}
$$

on $V^{\otimes 3}$, where $R_{i j}(\lambda)$ is the linear operator acting on the tensor product of the $i$-th and the $j$-th component of $V^{\otimes 3}$ as $R(\lambda)$.

For $x=\left(x_{1}, \ldots, x_{N}\right) \in\left(\mathbb{C}^{\times}\right)^{N}$ we define the reflection operator $T(x) \in \operatorname{End}(V)$ by

$$
T(x)\left(v_{a}\right):=x_{a}^{-1} v_{\bar{a}}, \quad T(x)\left(v_{\bar{a}}\right):=x_{a} v_{a} \quad(1 \leq a \leq N)
$$

and set

$$
K(\lambda \mid x, \beta):=\frac{\lambda T(x)+\beta}{\lambda+\beta} .
$$

Then the operator $K(\lambda \mid x, \beta)$ satisfies the boundary Yang-Baxter equation:

$$
\begin{aligned}
& R_{12}\left(\lambda_{1}-\lambda_{2}\right) K_{1}\left(\lambda_{1} \mid x, \beta\right) R_{21}\left(\lambda_{1}+\lambda_{2}\right) K_{2}\left(\lambda_{2} \mid x, \beta\right) \\
& \quad=K_{2}\left(\lambda_{2} \mid x, \beta\right) R_{21}\left(\lambda_{1}+\lambda_{2}\right) K_{1}\left(\lambda_{1} \mid x, \beta\right) R_{12}\left(\lambda_{1}-\lambda_{2}\right)
\end{aligned}
$$

on $V^{\otimes 2}$, where $K_{j}(\lambda \mid x, \beta)$ is the linear operator acting on the $j$-th component of $V^{\otimes 2}$ as $K(\lambda \mid x, \beta)$.

For $x=\left(x_{1}, \ldots, x_{N}\right) \in\left(\mathbb{C}^{\times}\right)^{N}$ and $y=\left(y_{1}, \ldots, y_{n}\right) \in \mathbb{C}^{n}$, we define the linear operator $Q_{m}(x \mid y)(1 \leq m \leq n)$ acting on $V^{\otimes n}$ by

$$
\begin{aligned}
Q_{m}(x \mid y):= & R_{m, m-1}\left(y_{m}-y_{m-1}-c\right) \cdots R_{m, 1}\left(y_{m}-y_{1}-c\right) K_{m}\left(y_{m}-c / 2 \mid x, \beta\right) \\
& \times R_{1, m}\left(y_{1}+y_{m}\right) \cdots R_{m-1, m}\left(y_{m-1}+y_{m}\right) \\
& \times R_{m, m+1}\left(y_{m}+y_{m+1}\right) \cdots R_{m, n}\left(y_{m}+y_{n}\right) \\
& \times K_{m}\left(y_{m} \mid \underline{1}, \alpha\right) R_{m, n}\left(y_{m}-y_{n}\right) \cdots R_{m, m+1}\left(y_{m}-y_{m+1}\right),
\end{aligned}
$$

where $\underline{1}:=(1, \ldots, 1)$ and the lower indices of $R$ and $K$ in the right hand side signify the position of the components in $V^{\otimes n}$ on which they act.

Proposition 2.1. For $1 \leq l, m \leq n$ we have

$$
Q_{m}\left(x \mid \ldots, y_{l}-c, \ldots\right) Q_{l}(x \mid y)=Q_{l}\left(x \mid \ldots, y_{m}-c, \ldots\right) Q_{m}(x \mid y) .
$$

Proof. It follows from (2.1) and (2.3).

Let $f(x \mid y)$ be a function on $\left(\mathbb{C}^{\otimes N}\right) \times \mathbb{C}^{n}$ taking values in $V^{\otimes n}$. We denote by $\Delta_{m}(1 \leq m \leq n)$ the shift operator with respect to $y_{m}$ :

$$
\Delta_{m} f(x \mid y):=f\left(x \mid y_{1}, \ldots, y_{m}-c, \ldots, y_{n}\right) .
$$


From Proposition 2.1 the following system of difference equations is consistent:

$$
\Delta_{m} f(x \mid y)=Q_{m}(x \mid y) f(x \mid y) \quad(m=1, \ldots, n) .
$$

Definition 2.2. We call the system (2.4) of difference equations the boundary rational $q K Z$ equation.

\section{Compatible differential equations}

3.1. Commuting differential operators. We denote by $e_{a b} \in \operatorname{End}(V)(a, b \in$ $\{1, \ldots, N, \overline{1}, \ldots, \bar{N}\})$ the matrix unit acting by $e_{a b} v_{p}=\delta_{b p} v_{a}$. In this section, for $u \in \operatorname{End}(V)$ and $1 \leq j \leq n$, we denote by $u^{(j)} \in \operatorname{End}\left(V^{\otimes n}\right)$ the linear operator acting on the $j$-th component of $V^{\otimes n}$ as $u$. For $1 \leq a, b \leq N$ we set

$$
E_{a b}:=e_{a b}+e_{\bar{a} \bar{b}}, \quad \bar{E}_{a b}:=e_{a \bar{b}}+e_{\bar{a} b},
$$

and define $\mathbf{X}_{a b}, \mathbf{Y}_{a b}, \mathbf{Z}_{a b} \in \operatorname{End}\left(V^{\otimes n}\right)$ by

$$
\begin{aligned}
\mathbf{X}_{a b} & :=\sum_{1 \leq i<j \leq n}\left(e_{a b}^{(i)} E_{b a}^{(j)}+e_{\bar{b} \bar{a}}^{(i)} E_{a b}^{(j)}\right), \\
\mathbf{Y}_{a b} & :=\sum_{1 \leq i<j \leq n}\left(e_{a \bar{b}}^{(i)} \bar{E}_{b a}^{(j)}+e_{b \bar{a}}^{(i)} \bar{E}_{a b}^{(j)}\right), \\
\mathbf{Z}_{a b} & :=\sum_{1 \leq i<j \leq n}\left(e_{\bar{a} b}^{(i)} \bar{E}_{b a}^{(j)}+e_{\bar{b} a}^{(i)} \bar{E}_{a b}^{(j)}\right) .
\end{aligned}
$$

Note that $\mathbf{Y}_{a b}=\mathbf{Y}_{b a}$ and $\mathbf{Z}_{a b}=\mathbf{Z}_{b a}$.

Define the linear operators $A_{a}(y)$ and $B_{a}(x)(1 \leq a \leq N)$ on $V^{\otimes n}$ by

$$
A_{a}(y):=\sum_{j=1}^{n} y_{j}\left(e_{a a}^{(j)}-e_{\overline{a a}}^{(j)}\right)+2 \alpha \sum_{j=1}^{n} e_{a \bar{a}}^{(j)}+k\left(-\sum_{p=1}^{a-1} \mathbf{X}_{p a}+\sum_{p=a+1}^{N} \mathbf{X}_{a p}+\sum_{p=1}^{N} \mathbf{Y}_{a p}\right)
$$

and

$$
\begin{aligned}
B_{a}(x) & :=2 \frac{\alpha+\beta x_{a}}{x_{a}^{2}-1} \sum_{j=1}^{n} \bar{E}_{a a}^{(j)}+k\left\{\sum_{p=1}^{a-1} \frac{x_{a}}{x_{a}-x_{p}}\left(\mathbf{X}_{a p}+\mathbf{X}_{p a}\right)\right. \\
& \left.+\sum_{p=a+1}^{N} \frac{x_{p}}{x_{a}-x_{p}}\left(\mathbf{X}_{a p}+\mathbf{X}_{p a}\right)+\sum_{p=1}^{N} \frac{1}{x_{a} x_{p}-1}\left(\mathbf{Y}_{a p}+\mathbf{Z}_{a p}\right)\right\}
\end{aligned}
$$

Set

$$
L_{a}(x \mid y):=A_{a}(y)+B_{a}(x) \quad(1 \leq a \leq N) .
$$

By direct calculation we can check the commutativity:

Lemma 3.1. For $1 \leq a, b \leq N$ we have

$$
\left[A_{a}(y), A_{b}(y)\right]=0, \quad\left[L_{a}(x \mid y), L_{b}(x \mid y)\right]=0 .
$$


Now define the differential operators $D_{a}(x \mid y)(1 \leq a \leq N)$ by

$$
D_{a}(x \mid y):=c x_{a} \frac{\partial}{\partial x_{a}}+L_{a}(x \mid y) .
$$

Proposition 3.2. The differential operators $D_{a}(x \mid y)(a=1, \ldots, N)$ commute with each other.

Proof. It follows from Lemma 3.1 and the equality $x_{a} \frac{\partial L_{b}}{\partial x_{a}}=x_{b} \frac{\partial L_{a}}{\partial x_{b}}$ for $1 \leq a, b \leq$ $N$.

3.2. Compatibility. In this subsection we prove the main theorem:

Theorem 3.3. For $1 \leq a \leq N$ and $1 \leq m \leq n$ we have

$$
\left[D_{a}(x \mid y), \Delta_{m}^{-1} Q_{m}(x \mid y)\right]=0 .
$$

Hence the system of equations

$$
\begin{cases}\Delta_{m} f(x \mid y)=Q_{m}(x \mid y) f(x \mid y) & (1 \leq m \leq n) \\ D_{a}(x \mid y) f(x \mid y)=0 & (1 \leq a \leq N)\end{cases}
$$

is compatible.

To prove Theorem 3.3 we rewrite the linear operators $L_{a}(x \mid y)(1 \leq a \leq N)$ as follows. For $\lambda \in \mathbb{C}^{\times}$and $\gamma \in \mathbb{C}$, we define $I_{a}(\lambda \mid \gamma) \in \operatorname{End}(V)$ by

$$
I_{a}(\lambda \mid \gamma):=\gamma\left(e_{a a}-e_{\overline{a a}}\right)+2 \frac{\alpha+\beta \lambda}{\lambda^{2}-1} e_{\bar{a} a}+2 \frac{\alpha+\beta \lambda^{-1}}{1-\lambda^{-2}} e_{a \bar{a}} \quad(1 \leq a \leq N) .
$$

For $x=\left(x_{1}, \ldots, x_{N}\right) \in\left(\mathbb{C}^{\times}\right)^{n}$ we define $M_{a}(x) \in \operatorname{End}\left(V^{\otimes 2}\right)(1 \leq a \leq N)$ by

$$
\begin{aligned}
M_{a}(x) & :=\frac{2 k}{x_{a}-x_{a}^{-1}}\left(x_{a} e_{a \bar{a}}+x_{a}^{-1} e_{\bar{a} a}\right) \otimes\left(e_{a \bar{a}}+e_{\bar{a} a}\right) \\
& +k \sum_{\substack{p=1 \\
p \neq a}}^{N}\left(\frac{x_{a}}{x_{a}-x_{p}} U_{a p}+\frac{x_{p}}{x_{a}-x_{p}} U_{p a}+\frac{x_{a} x_{p}}{x_{a} x_{p}-1} J_{a p}+\frac{1}{x_{a} x_{p}-1} K_{a p}\right),
\end{aligned}
$$

where

$$
\begin{aligned}
U_{a b} & :=e_{a b} \otimes E_{b a}+e_{\bar{b} \bar{a}} \otimes E_{a b}, \quad J_{a b}:=e_{a \bar{b}} \otimes \bar{E}_{b a}+e_{b \bar{a}} \otimes \bar{E}_{a b}, \\
K_{a b} & :=e_{\bar{a} b} \otimes \bar{E}_{b a}+e_{\bar{b} a} \otimes \bar{E}_{a b} .
\end{aligned}
$$

Note that $J_{a b}=J_{b a}$ and $K_{a b}=K_{b a}$. Then we have

$$
L_{a}(x \mid y)=\sum_{j=1}^{n} I_{a}\left(x_{a} \mid y_{j}\right)^{(j)}+\sum_{1 \leq i<j \leq n} M_{a}(x)^{(i, j)}
$$

for $1 \leq a \leq N$, where $M_{a}(x)^{(i, j)}$ is the linear operator acting on the tensor product of the $i$-th and the $j$-th component of $V^{\otimes n}$ as $M_{a}(x)$. 
Lemma 3.4. For $x=\left(x_{1}, \ldots, x_{N}\right)$ and $1 \leq a \leq N$ we have

$$
\begin{aligned}
& R_{12}\left(y_{1}-y_{2}\right)\left(I_{a}\left(x_{a} \mid y_{1}\right)^{(1)}+I_{a}\left(x_{a} \mid y_{2}\right)^{(2)}+M_{a}(x)^{(1,2)}\right) R_{12}\left(y_{1}-y_{2}\right)^{-1} \\
& =I_{a}\left(x_{a} \mid y_{1}\right)^{(1)}+I_{a}\left(x_{a} \mid y_{2}\right)^{(2)}+M_{a}(x)^{(2,1)}
\end{aligned}
$$

on $V^{\otimes 2}$, where $M_{a}(x)^{(2,1)}=P M_{a}(x) P$.

Proof. Note that if $h \in \operatorname{End}\left(V^{\otimes 2}\right)$ is symmetric, i.e. $P h P=h$, then $h$ commutes with the $R$-matrix. We extract symmetric parts from the operator $I_{a}\left(x_{a} \mid y_{1}\right)^{(1)}+$ $I_{a}\left(x_{a} \mid y_{2}\right)^{(2)}+M_{a}(x)^{(1,2)}$ as follows. In the following we enclose symmetric parts in a square bracket [ ]. First we have

$$
\begin{aligned}
& I_{a}\left(x_{a} \mid y_{1}\right)^{(1)}+I_{a}\left(x_{a} \mid y_{2}\right)^{(2)} \\
& =\left[y_{1} \sum_{j=1}^{2}\left(e_{a a}^{(j)}-e_{\bar{a} \bar{a}}^{(j)}\right)+2 \frac{\alpha+\beta x_{a}}{x_{a}^{2}-1} \sum_{j=1}^{2} e_{\bar{a} a}^{(j)}+2 \frac{\alpha+\beta x_{a}^{-1}}{1-x_{a}^{-2}} \sum_{j=1}^{2} e_{a \bar{a}}^{(j)}\right] \\
& +\left(y_{2}-y_{1}\right)\left(e_{a a}^{(2)}-e_{\bar{a} \bar{a}}^{(2)}\right) .
\end{aligned}
$$

Note that $U_{a p}+U_{p a}$ and $J_{a p}+K_{a p}$ are symmetric. Using

$$
\begin{aligned}
U_{a p} & =\left[e_{\bar{p} \bar{a}}^{(1)} e_{a p}^{(2)}+e_{a p}^{(1)} e_{\bar{p} \bar{a}}^{(2)}\right]+e_{\bar{p} \bar{a}}^{(1)} e_{\bar{a} \bar{p}}^{(2)}+e_{a p}^{(1)} e_{p a}^{(2)}, \\
J_{a p} & =\left[e_{p \bar{a}}^{(1)} e_{a \bar{p}}^{(2)}+e_{a \bar{p}}^{(1)} e_{p \bar{a}}^{(2)}\right]+e_{p \bar{a}}^{(1)} e_{\bar{a} p}^{(2)}+e_{a \bar{p}}^{(1)} e_{\bar{p} a}^{(2)},
\end{aligned}
$$

we divide $M_{a}(x)^{(1,2)}$ as

$$
\begin{aligned}
& M_{a}(x)^{(1,2)}=\frac{2 k}{x_{a}-x_{a}^{-1}}\left[x_{a} e_{a \bar{a}}^{(1)} e_{a \bar{a}}^{(2)}+x_{a}^{-1} e_{\bar{a} a}^{(1)} e_{\bar{a} a}^{(2)}+x_{a}^{-1}\left(e_{a \bar{a}}^{(1)} e_{\bar{a} a}^{(2)}+e_{\bar{a} a}^{(1)} e_{a \bar{a}}^{(2)}\right)\right] \\
& +k \sum_{\substack{p=1 \\
p \neq a}}^{N}\left[\frac{x_{p}}{x_{a}-x_{p}}\left(U_{a p}+U_{p a}\right)+\frac{1}{x_{a} x_{p}-1}\left(J_{a p}+K_{a p}\right)\right] \\
& +k\left[\sum_{\substack{p=1 \\
p \neq a}}^{N}\left(e_{\bar{p} \bar{a}}^{(1)} e_{a p}^{(2)}+e_{a p}^{(1)} e_{\bar{p} \bar{a}}^{(2)}+e_{p \bar{a}}^{(1)} e_{a \bar{p}}^{(2)}+e_{a \bar{p}}^{(1)} e_{p \bar{a}}^{(2)}\right)-\left(e_{a a}^{(1)} e_{a a}^{(2)}+e_{\bar{a} \bar{a}}^{(1)} e_{\bar{a} \bar{a})}^{(2)}\right)\right] \\
& +k \sum_{p}^{\prime}\left(e_{a p}^{(1)} e_{p a}^{(2)}+e_{p \bar{a}}^{(1)} e_{\bar{a} \bar{p}}^{(2)}\right),
\end{aligned}
$$

where $\sum_{p}^{\prime}$ is a sum over all indices $p \in\{1, \ldots, N, \overline{1}, \ldots, \bar{N}\}$. Thus we find

$$
\begin{aligned}
& I_{a}\left(x_{a} \mid y_{1}\right)^{(1)}+I_{a}\left(x_{a} \mid y_{2}\right)^{(2)}+M_{a}(x)^{(1,2)} \\
& =[(\text { symmetric part })]+\left(y_{2}-y_{1}\right)\left(e_{a a}^{(2)}-e_{\bar{a} \bar{a}}^{(2)}\right)+k \sum_{p}^{\prime}\left(e_{a p}^{(1)} e_{p a}^{(2)}+e_{p \bar{a}}^{(1)} e_{\bar{a} p}^{(2)}\right) .
\end{aligned}
$$


Now we make use of the intertwining property of the $R$-matrix:

$$
\begin{aligned}
& R(\lambda)\left(\lambda \cdot 1 \otimes e_{l m}+k \sum_{p}^{\prime} e_{p m} \otimes e_{l p}\right)=\left(\lambda \cdot 1 \otimes e_{l m}+k \sum_{p}^{\prime} e_{l p} \otimes e_{p m}\right) R(\lambda), \\
& R(\lambda)\left(\lambda \cdot 1 \otimes e_{l m}-k \sum_{p}^{\prime} e_{l p} \otimes e_{p m}\right)=\left(\lambda \cdot 1 \otimes e_{l m}-k \sum_{p}^{\prime} e_{p m} \otimes e_{l p}\right) R(\lambda)
\end{aligned}
$$

for any $l, m \in\{1, \ldots, N, \overline{1}, \ldots, \bar{N}\}$. Then we see that

$$
\begin{aligned}
& R_{12}\left(y_{1}-y_{2}\right)\left(\left(y_{2}-y_{1}\right)\left(e_{a a}^{(2)}-e_{\bar{a} \bar{a}}^{(2)}\right)+k \sum_{p}^{\prime}\left(e_{a p}^{(1)} e_{p a}^{(2)}+e_{p \bar{a}}^{(1)} e_{\bar{a} p}^{(2)}\right)\right) R_{12}\left(y_{1}-y_{2}\right)^{-1} \\
& =\left(y_{2}-y_{1}\right)\left(e_{a a}^{(2)}-e_{\bar{a} \bar{a}}^{(2)}\right)+k \sum_{p}^{\prime}\left(e_{p a}^{(1)} e_{a p}^{(2)}+e_{\bar{a} p}^{(1)} e_{p \bar{a}}^{(2)}\right) \\
& =\left(y_{2}-y_{1}\right)\left(e_{a a}^{(2)}-e_{\bar{a} \bar{a}}^{(2)}\right)+2 k e_{\bar{a} a}^{(1)} e_{a \bar{a}}^{(2)} \\
& +k\left\{\sum_{\substack{p=1 \\
p \neq a}}^{N}\left(e_{p a}^{(1)} e_{a p}^{(2)}+e_{\bar{p} a}^{(1)} e_{a \bar{p}}^{(2)}+e_{\bar{a} p}^{(1)} e_{p \bar{a}}^{(2)}+e_{\bar{a} \bar{p}}^{(1)} e_{\bar{a} \bar{a}}^{(2)}\right)+\left(e_{a a}^{(1)} e_{a a}^{(2)}+e_{\bar{a} \bar{a}}^{(1)} e_{\bar{a} \bar{a})}^{(2)}\right)\right\} .
\end{aligned}
$$

Adding this to the symmetric part in $(3.3)$ we obtain $I_{a}\left(x_{a} \mid y_{1}\right)^{(1)}+I_{a}\left(x_{a} \mid y_{2}\right)^{(2)}+$ $M_{a}(x)^{(2,1)}$.

Proof of Theorem 3.3. We split the operator $Q_{m}(x \mid y)$ into three parts:

$$
Q_{m}(x \mid y)=Q_{m}^{\prime}(y) K_{m}\left(y_{m}-c / 2 \mid x, \beta\right) Q_{m}^{\prime \prime}(y),
$$

where

$$
Q_{m}^{\prime}(y):=R_{m, m-1}\left(y_{m}-y_{m-1}-c\right) \cdots R_{m, 1}\left(y_{m}-y_{1}-c\right)
$$

and $Q_{m}^{\prime \prime}(y)$ is determined by (3.4) $)$. For $g \in \mathrm{GL}\left(V^{\otimes n}\right)$ we denote by $\operatorname{Ad}(g)$ the adjoint action $h \mapsto g h g^{-1}$ on $\operatorname{End}\left(V^{\otimes n}\right)$. Then the equality (3.1) is equivalent to

$$
\begin{aligned}
& c x_{a}\left(\frac{\partial}{\partial x_{a}} K_{m}\left(y_{m}-c / 2 \mid x, \beta\right)\right) K_{m}\left(y_{m}-c / 2 \mid x, \beta\right)^{-1} \\
& +\operatorname{Ad}\left(Q_{m}^{\prime}(y)\right)\left(L_{a}\left(x \mid \ldots, y_{m}-c, \ldots\right)\right) \\
& -\operatorname{Ad}\left(K_{m}\left(y_{m}-c / 2 \mid x, \beta\right) Q_{m}^{\prime \prime}(y)\right)\left(L_{a}\left(x \mid \ldots, y_{m}, \ldots\right)\right)=0 .
\end{aligned}
$$

The first term of (3.5) is equal to

$$
\frac{c\left(y_{m}-c / 2\right)}{\left(y_{m}-c / 2\right)^{2}-\beta^{2}}\left\{\left(y_{m}-c / 2\right)\left(e_{a a}^{(m)}-e_{\bar{a} \bar{a}}^{(m)}\right)-\beta\left(x_{a} e_{a \bar{a}}^{(m)}-x_{a}^{-1} e_{\bar{a} a}^{(m)}\right)\right\} .
$$


From Lemma 3.4 we see that the second term is equal to

$$
\sum_{\substack{j=1 \\ j \neq m}}^{n} I_{a}\left(x_{a} \mid y_{j}\right)^{(j)}+I_{a}\left(x_{a} \mid y_{m}-c\right)^{(m)}+\sum_{\substack{j=1 \\ j \neq m}}^{n} M_{a}(x)^{(m, j)}+\sum_{\substack{1 \leq i<j \leq n \\ i, j \neq m}}^{n} M_{a}(x)^{(i, j)} .
$$

Let us calculate the third term. Using Lemma 3.4 and

$$
\begin{aligned}
& \operatorname{Ad}(K(\gamma \mid \underline{1}, \alpha))\left(I_{a}(\lambda \mid \gamma)\right)=I_{a}(\lambda \mid-\gamma), \\
& \operatorname{Ad}\left(K_{2}(\gamma \mid \underline{1}, \alpha)\right)\left(M_{a}(x)^{(1,2)}\right)=M_{a}(x)^{(1,2)},
\end{aligned}
$$

we obtain

$$
\begin{aligned}
& \operatorname{Ad}\left(Q_{m}^{\prime \prime}(y)\right)\left(L_{a}(x \mid y)\right) \\
& =\sum_{\substack{j=1 \\
j \neq m}}^{n} I_{a}\left(x_{a} \mid y_{j}\right)^{(j)}+I_{a}\left(x_{a} \mid-y_{m}\right)^{(m)}+\sum_{\substack{j=1 \\
j \neq m}}^{n} M_{a}(x)^{(m, j)}+\sum_{\substack{1 \leq i<j \leq n \\
i, j \neq m}}^{n} M_{a}(x)^{(i, j)} .
\end{aligned}
$$

What remains is to calculate the image of the second and the third term above by the operator $\operatorname{Ad}\left(K_{m}\left(y_{m}-c / 2 \mid x, \beta\right)\right)$. By direct calculation we find

$$
\begin{aligned}
& \operatorname{Ad}(K(\gamma-c / 2 \mid x, \beta))\left(I_{a}\left(x_{a} \mid-\gamma\right)\right) \\
& =I_{a}\left(x_{a} \mid \gamma\right)+\frac{c \beta}{(\gamma-c / 2)^{2}-\beta^{2}}\left\{\beta\left(e_{a a}-e_{\bar{a} \bar{a}}\right)+(\gamma-c / 2)\left(x_{a}^{-1} e_{\bar{a} a}-x_{a} e_{a \bar{a}}\right)\right\}
\end{aligned}
$$

and

$$
\operatorname{Ad}\left(K_{1}(\gamma \mid x, \beta)\right)\left(M_{a}(x)^{(1,2)}\right)=M_{a}(x)^{(1,2)} .
$$

Using these formulas we see that the third term of (3.5) is equal to

$$
\begin{aligned}
(-1) \times( & \sum_{j=1}^{n} I_{a}\left(x_{a} \mid y_{j}\right)^{(j)}+\sum_{\substack{j=1 \\
j \neq m}}^{n} M_{a}(x)^{(m, j)}+\sum_{\substack{1 \leq i<j \leq n \\
i, j \neq m}}^{n} M_{a}(x)^{(i, j)} \\
& \left.+\frac{c \beta}{\left(y_{m}-c / 2\right)^{2}-\beta^{2}}\left\{\beta\left(e_{a a}^{(m)}-e_{\bar{a} \bar{a}}^{(m)}\right)+\left(y_{m}-c / 2\right)\left(x_{a}^{-1} e_{\bar{a} a}^{(m)}-x_{a} e_{a \bar{a}}^{(m)}\right)\right\}\right) .
\end{aligned}
$$

The sum of (3.6), (3.7) and (3.8) is zero and this completes the proof.

\section{The Bispectral QKZ EQUation and its DEgeneration}

4.1. The double affine Hecke algebra of type $\left(C_{n}^{\vee}, C_{n}\right)$. Here we recall the definition and some properties of the double affine Hecke algebra of type $\left(C_{n}^{\vee}, C_{n}\right)$ [14. We denote by $\mathbb{F}:=\mathbb{C}\left(q^{1 / 2}, t^{1 / 2}, t_{0}^{1 / 2}, t_{n}^{1 / 2}, u_{0}^{1 / 2}, u_{n}^{1 / 2}\right)$ the coefficient field.

Definition 4.1. The double affine Hecke algebra $\mathbb{H}$ of type $\left(C_{n}^{\vee}, C_{n}\right)$ is the unital associative $\mathbb{F}$-algebra generated by $X_{i}^{ \pm 1}(1 \leq i \leq n)$ and $T_{i}(0 \leq i \leq n)$ satisfying the following relations: 
(i) quadratic Hecke relations

$$
\left(T_{i}-t_{i}^{1 / 2}\right)\left(T_{i}+t_{i}^{-1 / 2}\right)=0 \quad(0 \leq i \leq n)
$$

where $t_{i}^{1 / 2}:=t^{1 / 2}$ for $1 \leq i<n$.

(ii) braid relations

$$
\begin{aligned}
& T_{i} T_{i+1} T_{i} T_{i+1}=T_{i+1} T_{i} T_{i+1} T_{i} \quad(i=0, n-1), \\
& T_{i} T_{i+1} T_{i}=T_{i+1} T_{i} T_{i+1} \quad(1 \leq i<n), \quad T_{i} T_{j}=T_{j} T_{i} \quad(|i-j| \geq 2) .
\end{aligned}
$$

(iii) relations between $X$ and $T$

$$
\begin{aligned}
& X_{i} X_{j}=X_{j} X_{i} \quad(\forall i, j), \quad T_{i} X_{j}=X_{j} T_{i} \quad(|i-j| \geq 2 \text { or }(i, j)=(n, n-1)), \\
& T_{i} X_{i} T_{i}=X_{i+1} \quad(1 \leq i \leq n-1), \quad X_{n}^{-1} T_{n}^{-1}=T_{n} X_{n}+\left(u_{n}^{1 / 2}-u_{n}^{-1 / 2}\right), \\
& q^{-1 / 2} T_{0}^{-1} X_{1}=q^{1 / 2} X_{1}^{-1} T_{0}+\left(u_{0}^{1 / 2}-u_{0}^{-1 / 2}\right) .
\end{aligned}
$$

Noumi found the polynomial representation of $\mathbb{H}$ given as follows [13]. Let $W=$ $\left\langle s_{0}, \ldots, s_{n}\right\rangle$ be the affine Weyl group of type $C_{n}$. The group $W$ acts on the Laurent polynomial ring $\mathbb{F}\left[X^{ \pm 1}\right]=\mathbb{F}\left[X_{1}^{ \pm 1}, \ldots, X_{n}^{ \pm 1}\right]$ by

$$
\begin{aligned}
\left(s_{0} f\right)(X) & =f\left(q X_{1}^{-1}, X_{2}, \ldots, X_{n}\right), \\
\left(s_{i} f\right)(X) & =f\left(\ldots, X_{i+1}, X_{i}, \ldots\right) \quad(1 \leq i<n), \\
\left(s_{n} f\right)(X) & =f\left(X_{1}, \ldots, X_{n-1}, X_{n}^{-1}\right) .
\end{aligned}
$$

Define the $\mathbb{F}$-linear operators $\widehat{T}_{i}(0 \leq i \leq n)$ on $\mathbb{F}\left[X^{ \pm 1}\right]$ :

$$
\begin{aligned}
& \widehat{T}_{0}:=t_{0}^{1 / 2}+t_{0}^{-1 / 2} \frac{\left(1-q^{1 / 2} t_{0}^{1 / 2} u_{0}^{1 / 2} X_{1}^{-1}\right)\left(1+q^{1 / 2} t_{0}^{1 / 2} u_{0}^{-1 / 2} X_{1}^{-1}\right)}{1-q X_{1}^{-2}}\left(s_{0}-1\right), \\
& \widehat{T}_{i}:=t^{1 / 2}+t^{-1 / 2} \frac{1-t X_{i} / X_{i+1}}{1-X_{i} / X_{i+1}}\left(s_{i}-1\right) \quad(1 \leq i<n), \\
& \widehat{T}_{n}:=t_{n}^{1 / 2}+t_{n}^{-1 / 2} \frac{\left(1-t_{n}^{1 / 2} u_{n}^{1 / 2} X_{n}\right)\left(1+t_{n}^{1 / 2} u_{n}^{-1 / 2} X_{n}\right)}{1-X_{n}^{2}}\left(s_{n}-1\right) .
\end{aligned}
$$

Then the map $T_{i} \mapsto \widehat{T}_{i}$ and $X_{i} \mapsto X_{i}$ (left multiplication) gives a representation of $\mathbb{H}$ on $\mathbb{F}\left[X^{ \pm 1}\right]$. It is faithful and hence $\mathbb{H}$ is isomorphic to the $\mathbb{F}$-subalgebra of the smashed product algebra $\mathbb{F}(X) \# W$ generated by the difference operators $\widehat{T}_{i}(0 \leq$ $i \leq n$ ) and the multiplication operators $f(\underline{\mathrm{X}}) \in \mathbb{F}\left[X^{ \pm 1}\right]$ (see, e.g., section 2.1 in [11] for the definition of the smashed product algebra). Hereafter we identify $\mathbb{H}$ with the subalgebra of $\mathbb{F}(X) \# W$.

The subalgebra $H_{0}$ generated by $T_{i}(1 \leq i \leq n)$ is isomorphic to the Hecke algebra of type $C_{n}$. Denote by $W_{0}:=\left\langle s_{1}, \ldots, s_{n}\right\rangle$ the finite Weyl group of type $C_{n}$. Let $w=s_{j_{1}} \cdots s_{j_{r}}$ be a reduced expression of $w \in W_{0}$. Then we define $T_{w} \in H_{0}$ by $T_{w}:=T_{j_{1}} \cdots T_{j_{r}}$. The set $\left\{T_{w}\right\}_{w \in W_{0}}$ gives a basis of $H_{0}$. 
Set

They satisfy

$$
Y_{i}:=T_{i} \cdots T_{n-1}\left(T_{n} \cdots T_{0}\right) T_{1}^{-1} \cdots T_{i-1}^{-1} \quad(1 \leq i \leq n)
$$

$$
\begin{aligned}
& Y_{i} Y_{j}=Y_{j} Y_{i} \quad(\forall i, j), \quad T_{i} Y_{j}=Y_{j} T_{i} \quad(|i-j| \geq 2 \text { or }(i, j)=(n, n-1)) \\
& T_{i} Y_{i+1} T_{i}=Y_{i} \quad(1 \leq i \leq n-1), \quad T_{n}^{-1} Y_{n}=Y_{n}^{-1} T_{n}+\left(t_{0}^{1 / 2}-t_{0}^{-1 / 2}\right)
\end{aligned}
$$

and

$$
q^{-1 / 2} Y_{1}^{-1} U_{n}^{-1}=q^{1 / 2} U_{n} Y_{1}+\left(u_{0}^{1 / 2}-u_{0}^{-1 / 2}\right),
$$

where $U_{n}:=X_{1}^{-1} T_{0} Y_{1}^{-1}$. The subalgebra $H$ generated by $T_{i}(1 \leq i \leq n)$ and $Y_{i}^{ \pm 1}(1 \leq i \leq n)$ is called the affine Hecke algebra of type $C_{n}$.

Let $*: \mathbb{F} \rightarrow \mathbb{F}$ be the $\mathbb{C}$-algebra involution defined by $\left(t_{0}^{1 / 2}\right)^{*}=u_{n}^{1 / 2}$ and the other parameters $q^{1 / 2}, t^{1 / 2}, t_{n}^{1 / 2}, u_{0}^{1 / 2}$ are fixed. It uniquely extends to the $\mathbb{C}$-algebra anti-involution on $\mathbb{H}$ such that

$$
T_{0}^{*}=U_{n}, \quad T_{i}^{*}=T_{i}, \quad X_{i}^{*}=Y_{i}^{-1}, \quad Y_{i}^{*}=X_{i}^{-1} \quad(1 \leq i \leq n) .
$$

The anti-involution $*$ is called duality anti-involution. For a Laurent polynomial $f$ of $n$-variables with the coefficients in $\mathbb{F}$, we define $f^{\diamond}$ by the equality $f^{\diamond}(Y)=(f(X))^{*}$.

Define the elements $\widetilde{S}_{i}(0 \leq i \leq n)$ in $\mathbb{F}(X) \# W$ by

$$
\begin{aligned}
\widetilde{S}_{0} & :=t_{0}^{-1 / 2}\left(1-q^{1 / 2} t_{0}^{1 / 2} u_{0}^{1 / 2} X_{1}^{-1}\right)\left(1+q^{1 / 2} t_{0}^{1 / 2} u_{0}^{-1 / 2} X_{1}^{-1}\right) s_{0}, \\
\widetilde{S}_{i} & :=t^{-1 / 2}\left(1-t X_{i} / X_{i+1}\right) s_{i} \quad(1 \leq i<N), \\
\widetilde{S}_{n} & :=t_{n}^{-1 / 2}\left(1-t_{n}^{1 / 2} u_{n}^{1 / 2} X_{n}\right)\left(1+t_{n}^{1 / 2} u_{n}^{-1 / 2} X_{n}\right) s_{n} .
\end{aligned}
$$

In fact they belong to the subalgebra $\mathbb{H}$. For example we have

$$
\widetilde{S}_{0}=\left(1-q X_{1}^{-2}\right) T_{0}-\left(t_{0}^{1 / 2}-t_{0}^{-1 / 2}\right)-\left(u_{0}^{1 / 2}-u_{0}^{-1 / 2}\right) q^{1 / 2} X_{1}^{-1} \in \mathbb{H} .
$$

The elements $\widetilde{S}_{i}$ and their dual $\widetilde{S}_{i}^{*}(0 \leq i \leq n)$ will play a fundamental role in the construction of the bispectral qKZ equation.

4.2. The bispectral $\mathbf{q K Z}$ equation. Here we construct the bispectral qKZ equation of type $\left(C_{n}^{\vee}, C_{n}\right)$. See [11] for the details in the case of type $G L_{n}$.

Hereafter we set the parameters $q^{1 / 2}, t^{1 / 2}, \ldots$ to generic complex values and consider $\mathbb{H}$ as a $\mathbb{C}$-algebra. Then we have the Poincaré-Birkhoff-Witt (PBW) decomposition of $H_{0}$ and $\mathbb{H}$ as $\mathbb{C}$-vector spaces:

$$
H \simeq H_{0} \otimes \mathbb{C}\left[Y^{ \pm 1}\right], \quad \mathbb{H} \simeq \mathbb{C}\left[X^{ \pm 1}\right] \otimes H_{0} \otimes \mathbb{C}\left[Y^{ \pm 1}\right]
$$

Set $\mathbb{L}:=\mathbb{C}\left[X^{ \pm 1}\right] \otimes \mathbb{C}\left[Y^{ \pm 1}\right]$. The DAHA $\mathbb{H}$ has $\mathbb{L}$-module structure defined by

$$
(f \otimes g) . h=f(x) h g(y) \quad(f \otimes g \in \mathbb{L}, h \in \mathbb{H}) .
$$

We consider $\mathbb{L}$ as the ring of regular functions on $T \times T$, where $T$ is the $n$-dimensional torus $T:=\left(\mathbb{C}^{\times}\right)^{n}$. From the PBW decomposition, any element of $\mathbb{H}$ can be regarded as an $H_{0}$-valued regular function on $T \times T$. Let $\mathbb{K}$ be the field of meromorphic 
functions on $T \times T$. Then $H_{0}^{\mathbb{K}}:=\mathbb{K} \otimes_{\mathbb{L}} \mathbb{H}$ is a vector space of $H_{0}$-valued meromorphic functions on $T \times T$.

Denote the translations in $W$ by

$$
\epsilon_{i}:=s_{i} \cdots s_{n-1}\left(s_{n} \cdots s_{0}\right) s_{1} \cdots s_{i-1} \quad(1 \leq i \leq n) .
$$

Then $W$ is a semi-direct product $W \simeq W_{0} \ltimes \Gamma$ of the finite Weyl group $W_{0}:=$ $\left\langle s_{1}, \ldots, s_{n}\right\rangle$ and the lattice $\Gamma \simeq \mathbb{Z}^{n}$ generated by $\epsilon_{i}(1 \leq i \leq n)$. Define the involution $\diamond: W \rightarrow W$ by $w_{0}^{\diamond}=w_{0}$ for $w_{0} \in W_{0}$ and $\epsilon_{i}^{\diamond}=\epsilon_{i}^{-1}(1 \leq i \leq n)$. Then $W \times W$ acts on $\mathbb{L}$ by $\left(w, w^{\prime}\right)(f(X) \otimes g(Y))=(w f)(X) \otimes\left(w^{\prime \diamond} g\right)(Y)$. The action naturally extends to that on $\mathbb{K}$.

Let $w=s_{j_{1}} \cdots s_{j_{l}}$ be a reduced expression of $w \in W$. Then the element $\widetilde{S}_{w}:=$ $\widetilde{S}_{j_{1}} \cdots \widetilde{S}_{j_{l}} \in \mathbb{H}$ is well-defined. We denote by $d_{w}(X)$ the Laurent polynomial uniquely determined by the equality $\widetilde{S}_{w}=d_{w}(X) w$ in $\mathbb{F}(X) \# W$.

For $\left(w, w^{\prime}\right) \in W \times W$, consider the $\mathbb{C}$-linear endomorphism $\widetilde{\sigma}_{\left(w, w^{\prime}\right)}$ on $\mathbb{H}$ defined by

$$
\widetilde{\sigma}_{\left(w, w^{\prime}\right)}(h):=\widetilde{S}_{w} h \widetilde{S}_{w^{\prime}}^{*}
$$

Then we have

$$
\widetilde{\sigma}_{\left(w, w^{\prime}\right)}(f . h)=\left(w, w^{\prime}\right)(f) . \widetilde{\sigma}_{\left(w, w^{\prime}\right)}(h) \quad(f \in \mathbb{L}, h \in \mathbb{H}) .
$$

The map $\widetilde{\sigma}_{\left(w, w^{\prime}\right)}$ extends to the $\mathbb{C}$-linear endomorphism on $H_{0}^{\mathbb{K}}$ satisfying (4.5) for all $f \in \mathbb{K}$ and $h \in \mathbb{H}$.

Now we define $\tau\left(w, w^{\prime}\right) \in \operatorname{End}_{\mathbb{C}}\left(H_{0}^{\mathbb{K}}\right)\left(\left(w, w^{\prime}\right) \in W \times W\right)$ by

$$
\tau\left(w, w^{\prime}\right)(F):=d_{w}(X)^{-1} d_{w^{\prime}}^{\diamond}(Y)^{-1} \cdot \widetilde{\sigma}_{\left(w, w^{\prime}\right)}(F) \quad\left(F \in H_{0}^{\mathbb{K}}\right) .
$$

Then the bispectral $q K Z$ equation is given by

$$
\tau(\mu, \nu)(F)=F \quad(\forall \mu, \nu \in \Gamma) .
$$

Any element $F \in H_{0}^{\mathbb{K}}$ is written in the form $F=\sum_{w \in W_{0}} f_{w} \cdot T_{w}\left(f_{w} \in \mathbb{K}\right)$. Substituting it into (4.6) we have

$$
\sum_{w \in W_{0}}\left((\mu, \nu)\left(f_{w}\right) \cdot d_{\mu}(X)^{-1} d_{\nu}^{\diamond}(Y)^{-1}\right) \cdot\left(\widetilde{S}_{\mu} T_{w} \widetilde{S}_{\nu}^{*}\right)=\sum_{w \in W_{0}} f_{w} \cdot T_{w}
$$

This gives a system of $q$-difference equations for $\left\{f_{w}\right\}_{w \in H_{0}}$, which is an explicit form of the bispectral qKZ equation.

4.3. The degenerate double affine Hecke algebra. In this subsection we consider the trigonometric degeneration of the DAHA of type $\left(C_{n}^{\vee}, C_{n}\right)$. We refer to [4] for the general theory on it.

In the following we make use of $X_{i}, T_{i}, Y_{i}(1 \leq i \leq n)$ as generators of $\mathbb{H}$. Note that $T_{0}$ is recovered from them by $T_{0}=T_{1}^{-1} \cdots T_{n-1}^{-1} \cdot T_{n}^{-1} \cdots T_{1}^{-1} Y_{1}$. Let $\hbar$ be a small 
parameter. We set

$$
\begin{aligned}
& q=e^{\hbar c / 2}, t=e^{\hbar k / 2}, t_{0}=e^{\hbar k_{0} / 2}, t_{n}=e^{\hbar k_{n} / 2}, u_{0}=e^{\hbar k_{0}^{*} / 2}, u_{n}=e^{\hbar k_{n}^{*} / 2}, \\
& X_{i}=x_{i}, \quad T_{i}=s_{i}+\hbar \widetilde{T}_{i}+o(\hbar), \quad Y_{i}=e^{\hbar y_{i}} \quad(1 \leq i \leq n) .
\end{aligned}
$$

and take the limit $\hbar \rightarrow 0$. In (4.9) we introduced accessory generators $\widetilde{T}_{i}(1 \leq$ $i \leq n)$ to avoid rewriting formulas in the form of Lusztig's relations. For example, substitute (4.8) and (4.9) into the relations

$$
\left(T_{i}-t^{1 / 2}\right)\left(T_{i}+t^{-1 / 2}\right)=0, \quad T_{i} Y_{i+1} T_{i}=Y_{i} \quad(1 \leq i \leq n)
$$

and expand them into power series of $\hbar$. Taking the zeroth and the first order terms we obtain

$$
s_{i}^{2}=1, \quad s_{i} \widetilde{T}_{i}+\widetilde{T}_{i} s_{i}=k s_{i}, \quad \widetilde{T}_{i} s_{i}+s_{i} y_{i+1} s_{i}+s_{i} \widetilde{T}_{i}=y_{i} .
$$

Eliminating $\widetilde{T}_{i}$, we find $y_{i} s_{i}=s_{i} y_{i+1}+k$. Thus we get closed relations among $x_{i}, s_{i}$ and $y_{i}(1 \leq i \leq n)$. The parameters $k_{0}, k_{n}, k_{0}^{*}$ and $k_{n}^{*}$ appear only in the form of $k_{0}+k_{n}$ and $k_{0}^{*}+k_{n}^{*}$. Setting $\alpha:=\left(k_{0}+k_{n}\right) / 2$ and $\beta:=\left(k_{0}^{*}+k_{n}^{*}\right) / 2$, we obtain the trigonometric degeneration of $\mathbb{H}$ :

Definition 4.2. The degenerate double affine Hecke algebra $\overline{\mathbb{H}}$ of type $\left(C_{n}^{\vee}, C_{n}\right)$ is the unital associative algebra generated by $x_{i}, s_{i}$ and $y_{i}(1 \leq i \leq n)$ satisfying the following relations:

$$
\begin{aligned}
& s_{i}^{2}=1 \quad(1 \leq i \leq n), \quad s_{i} s_{i+1} s_{i}=s_{i+1} s_{i} s_{i+1} \quad(1 \leq i<n), \\
& s_{n-1} s_{n} s_{n-1} s_{n}=s_{n} s_{n-1} s_{n} s_{n-1}, \\
& s_{i} x_{i} s_{i}=x_{i+1} \quad(1 \leq i<n), \quad s_{n} x_{n} s_{n}=x_{n}^{-1}, \\
& y_{i} s_{i}=s_{i} y_{i+1}+k \quad(1 \leq i<n), \quad y_{n} s_{n}=-s_{n} y_{n}+2 \alpha, \\
& {\left[s_{i}, x_{j}\right]=0=\left[\begin{array}{ll}
\left.s_{i}, y_{j}\right] \quad(|i-j|>1 \text { or }(i, j)=(n, n-1)), \\
k\left(\tilde{s}_{j i}-s_{j i}\right) x_{j} \\
c x_{i}+2\left(\alpha x_{i}^{-1}+\beta\right) r_{i} \\
+k\left(\sum_{1 \leq l<i} s_{l i} x_{l}+\sum_{i<l \leq n} s_{i l} x_{i}+\sum_{l(\neq i)} \tilde{s}_{i l} x_{i}\right) & (i=j) \\
k\left(\tilde{s}_{i j} x_{j}-s_{i j} x_{i}\right) & (i<j),
\end{array}\right.}
\end{aligned}
$$

where

$$
\begin{aligned}
& s_{i j}=s_{j i}:=\left(s_{i} \cdots s_{j-1}\right)\left(s_{j-2} \cdots s_{i}\right) \quad(i<j), \\
& r_{i}:=\left(s_{i} \cdots s_{n}\right)\left(s_{n-1} \cdots s_{i}\right) \quad(1 \leq i \leq n), \quad \tilde{s}_{i j}:=r_{i} r_{j} s_{i j} .
\end{aligned}
$$

The subalgebra generated by $s_{i}(1 \leq i<n)$ is isomorphic to the group algebra $\mathbb{C}\left[W_{0}\right]$. The subalgebra $\bar{H}$ generated by $s_{i}(1 \leq i<n)$ and $y_{i}(1 \leq i \leq n)$ is called the degenerate affine Hecke algebra of type $C_{n}$. The subalgebra generated by $x_{i}(1 \leq i \leq n)$ and $s_{i}(1 \leq i<n)$ is isomorphic to the group algebra $\mathbb{C}[W]$ of 
the affine Weyl group. We identify them through the isomorphism determined by $x_{1}^{-1} r_{1} \mapsto s_{0}$ and $s_{i} \mapsto s_{i}(1 \leq i \leq n)$. We have the PBW decomposition:

$$
\mathbb{C}[W] \simeq \mathbb{C}\left[x^{ \pm 1}\right] \otimes \mathbb{C}\left[W_{0}\right], \quad \bar{H} \simeq \mathbb{C}\left[W_{0}\right] \otimes \mathbb{C}[y], \quad \overline{\mathbb{H}} \simeq \mathbb{C}\left[x^{ \pm 1}\right] \otimes \mathbb{C}\left[W_{0}\right] \otimes \mathbb{C}[y],
$$

where $\mathbb{C}\left[x^{ \pm 1}\right]:=\mathbb{C}\left[x_{1}^{ \pm 1}, \ldots, x_{n}^{ \pm 1}\right]$ and $\mathbb{C}[y]:=\mathbb{C}\left[y_{1}, \ldots, y_{n}\right]$.

We regard $\overline{\mathbb{L}}:=\mathbb{C}\left[x^{ \pm 1}\right] \otimes \mathbb{C}[y]$ as the ring of regular functions on $\left(\mathbb{C}^{\times}\right)^{n} \times \mathbb{C}^{n}$. Denote by $\overline{\mathbb{K}}$ the field of meromorphic functions on $\left(\mathbb{C}^{\times}\right)^{n} \times \mathbb{C}^{n}$. We give $\overline{\mathbb{L}}$-module structure to $\overline{\mathbb{H}}$ in the same way as (4.4). Then $\mathbb{C}\left[W_{0}\right]^{\overline{\mathbb{K}}}:=\overline{\mathbb{K}} \otimes_{\overline{\mathbb{L}}} \overline{\mathbb{H}}$ is the vector space of $\mathbb{C}\left[W_{0}\right]$-valued meromorphic functions. Any element $G \in \mathbb{C}\left[W_{0}\right]^{\overline{\mathbb{K}}}$ is uniquely represented in the form $G=\sum_{w \in W_{0}} g_{w} . w$ where $g_{w} \in \overline{\mathbb{K}}$.

We define two maps

$$
\eta_{L}: \bar{H} \rightarrow \operatorname{End}_{\overline{\mathbb{K}}}\left(\mathbb{C}\left[W_{0}\right]^{\overline{\mathbb{K}}}\right), \quad \eta_{R}: \mathbb{C}[W] \rightarrow \operatorname{End}_{\overline{\mathbb{K}}}\left(\mathbb{C}\left[W_{0}\right]^{\overline{\mathbb{K}}}\right)
$$

by

$$
\eta_{L}(h)\left(\sum_{w \in W_{0}} g_{w} \cdot w\right):=\sum_{w \in W_{0}} g_{w} \cdot(h w), \quad \eta_{R}(\xi)\left(\sum_{w \in W_{0}} g_{w} \cdot w\right):=\sum_{w \in W_{0}} g_{w} \cdot(w \xi) .
$$

Then the map $\eta_{L}$ (resp. $\eta_{R}$ ) is an algebra (resp. anti-algebra) homomorphism. Hence they determine $(\bar{H}, \mathbb{C}[W])$-bimodule structure on $\mathbb{C}\left[W_{0}\right]^{\mathbb{K}}$.

4.4. Degeneration of the bispectral $\mathbf{q K Z}$ equation ( $Y$-side). First we consider a part of the bispectral qKZ equation $\tau\left(1, \epsilon_{m}\right)(F)=F(1 \leq m \leq n)$, which is explicitly given by

$$
\sum_{w \in W_{0}}\left(\left(1, \epsilon_{m}\right)\left(f_{w}\right) \cdot d_{\epsilon_{m}}^{\diamond}(Y)^{-1}\right) \cdot T_{w} \widetilde{S}_{\epsilon_{m}}^{*}=\sum_{w \in W_{0}} f_{w} \cdot T_{w}
$$

where $F=\sum_{w \in W_{0}} f_{w} \cdot T_{w}\left(f_{w} \in \mathbb{K}\right)$.

In the limit $\hbar \rightarrow 0$ we find

$$
\begin{aligned}
& d_{\epsilon_{m}}^{\diamond}(Y)=4 \hbar^{2 n} p_{m}^{(0)}(y)+o\left(\hbar^{2 n}\right), \\
& \widetilde{S}_{\epsilon_{m}}^{*}=-4 \hbar^{2 n} \tilde{\Psi}_{m}+o\left(\hbar^{2 n}\right),
\end{aligned}
$$

where the function $p_{m}^{(0)}(y)$ is defined by

$$
\begin{aligned}
p_{m}^{(0)}(y) & :=\left(y_{m}-\alpha\right)\left(y_{m}-\beta-c / 2\right) \\
& \times \prod_{j=1}^{m-1}\left(y_{m}-y_{j}-c-k\right) \prod_{j=m+1}^{n}\left(y_{m}-y_{j}-k\right) \prod_{j(\neq m)}\left(y_{m}+y_{j}-k\right) .
\end{aligned}
$$

The element $\tilde{\Psi}_{m}$ is given by

$$
\tilde{\Psi}_{m}:=\tilde{\tau}_{m-1} \cdots \tilde{\tau}_{1}\left(\tilde{\tau}_{0} \cdots \tilde{\tau}_{n}\right) \tilde{\tau}_{n-1} \cdots \tilde{\tau}_{m}
$$

where

$$
\tilde{\tau}_{0}:=x_{1}^{-1} r_{1}\left(y_{1}+c / 2\right)+\beta, \quad \tilde{\tau}_{i}:=s_{i}\left(y_{i}-y_{i+1}\right)-k(1 \leq i<n), \quad \tilde{\tau}_{n}:=s_{n} y_{n}-\alpha .
$$


Suppose that $F=F(X \mid Y) \in H_{0}^{\mathbb{K}}$ is expanded as

$$
F=\hbar^{M}(\bar{G}+o(1)) \quad(\hbar \rightarrow 0)
$$

for some $M \in \mathbb{Z}$ and $\bar{G} \in \mathbb{C}\left[W_{0}\right]^{\overline{\mathbb{K}}} \backslash\{0\}$. Then we have

$$
\left(1, \epsilon_{m}\right) F=\hbar^{M}\left(\Delta_{m} \bar{G}+o(1)\right) .
$$

Hence the trigonometric degeneration of (4.11) is given by

$$
\sum_{w \in W_{0}} \Delta_{m}\left(g_{w}\right) \cdot\left(w \tilde{\Psi}_{m}\right)=-p_{m}^{(0)}(y) \bar{G}
$$

where $\bar{G}=\sum_{w \in W_{0}} g_{w} \cdot w\left(g_{w} \in \overline{\mathbb{K}}\right)$.

We define the group action $W \rightarrow \mathrm{GL}_{\mathbb{C}}(\overline{\mathbb{L}})$ by $s_{j} \mapsto \sigma_{j}(0 \leq j \leq n)$, where

$$
\begin{aligned}
\left(\sigma_{0} f\right)(x \mid y) & :=f\left(x \mid-y_{1}-c, y_{2}, \ldots, y_{n}\right), \\
\left(\sigma_{i} f\right)(x \mid y) & :=f\left(x \mid \ldots, y_{i+1}, y_{i}, \ldots\right) \quad(1 \leq i<n), \\
\left(\sigma_{n} f\right)(x \mid y) & :=f\left(x \mid y_{1}, \ldots, y_{n-1},-y_{n}\right) .
\end{aligned}
$$

It naturally extends to the action on $\overline{\mathbb{K}}$. Consider the $\mathbb{C}$-linear map $\tilde{\tau}_{i}^{R}(0 \leq i \leq$ $n$ ) on $\overline{\mathbb{H}}$ defined by $\tilde{\tau}_{i}^{R}(h):=h \tilde{\tau}_{i}$. Then for any $f \in \overline{\mathbb{L}}$ and $h \in \overline{\mathbb{H}}$ we have $\tilde{\tau}_{i}^{R}(f . h)=\sigma_{i}(f) \cdot \tilde{\tau}_{i}^{R}(h)$. The map $\tilde{\tau}_{i}^{R}$ extends to the $\mathbb{C}$-linear map on $\mathbb{C}\left[W_{0}\right]^{\overline{\mathbb{K}}}$ satisfying $\tilde{\tau}_{i}^{R}(f \cdot G)=\sigma_{i}(f) \cdot \tilde{\tau}_{i}^{R}(G)$ for any $f \in \overline{\mathbb{K}}$ and $G \in \mathbb{C}\left[W_{0}\right]^{\overline{\mathbb{K}}}$.

Consider the left action $\nu: W \rightarrow \mathrm{GL}_{\mathbb{C}}\left(\mathbb{C}\left[W_{0}\right]^{\overline{\mathbb{K}}}\right)$ defined by

$$
\nu\left(s_{i}\right)\left(\sum_{w \in W_{0}} g_{w} . w\right):=\sum_{w \in W_{0}} \sigma_{i}\left(g_{w}\right) . w \quad(0 \leq i \leq n) .
$$

Note that $\nu\left(\epsilon_{m}\right)=\Delta_{m}$. Then the maps $\nu\left(s_{i}\right) \tilde{\tau}_{i}^{R}(0 \leq i \leq n)$ are $\overline{\mathbb{K}}$-linear and given in terms of $\eta_{R}$ by

$$
\begin{aligned}
& \nu\left(s_{0}\right) \tilde{\tau}_{0}^{R}=-\left(\left(y_{1}+c / 2\right) \eta_{R}\left(x_{1}^{-1} r_{1}\right)-\beta\right), \\
& \nu\left(s_{i}\right) \tilde{\tau}_{i}^{R}=-\left(\left(y_{i}-y_{i+1}\right) \eta_{R}\left(s_{i}\right)+k\right) \quad(1 \leq i<n), \\
& \nu\left(s_{n}\right) \tilde{\tau}_{n}^{R}=-\left(y_{n} \eta_{R}\left(s_{n}\right)+\alpha\right) .
\end{aligned}
$$

For simplicity's sake, we denote the sequence of indices in the right hand side of (4.12) by $\left(j_{1}, \ldots, j_{2 n}\right)=(m, \ldots, n-1, n, \ldots, 0,1, \ldots, m-1)$ fixing $m$. Set

$$
\zeta_{m, l}:=\nu\left(s_{j_{2 n}} \cdots s_{j_{l+1}}\right)\left(\nu\left(s_{j_{l}}\right) \tilde{\tau}_{j_{l}}^{R}\right) \nu\left(s_{j_{2 n}} \cdots s_{j_{l+1}}\right)^{-1} \quad(1 \leq l \leq 2 n)
$$

and $\Psi_{m}:=\zeta_{m, 1} \cdots \zeta_{m, 2 n}$. Then $\Psi_{m}$ is a $\overline{\mathbb{K}}$-linear map acting on $\mathbb{C}\left[W_{0}\right]^{\overline{\mathbb{K}}}$, and the operator of right multiplication with $\tilde{\Psi}_{m}$ on $\overline{\mathbb{H}}$ is equal to $\Delta_{m} \Psi_{m}$. Therefore the equation (4.14) is rewritten as

$$
\Delta_{m} \Psi_{m}(\bar{G})=-p_{m}^{(0)}(y) \bar{G}
$$

for $\bar{G} \in \mathbb{C}\left[W_{0}\right]^{\overline{\mathbb{K}}}$. 
4.5. Degeneration of the bispectral qKZ equation ( $X$-side). Next we consider the trigonometric degeneration of $\tau\left(\epsilon_{a}, 1\right)(F)=F(1 \leq a \leq n)$, which is explicitly given by

$$
\sum_{w \in W_{0}}\left(\left(\epsilon_{a}, 1\right)\left(f_{w}\right) \cdot d_{\epsilon_{a}}(X)^{-1}\right) \cdot \widetilde{S}_{\epsilon_{a}} T_{w}=\sum_{w \in W_{0}} f_{w} \cdot T_{w},
$$

where $F=\sum_{w \in W_{0}} f_{w} \cdot T_{w}\left(f_{w} \in \mathbb{K}\right)$.

By direct calculation we have

$$
d_{\epsilon_{a}}(X)=d_{\epsilon_{a}}^{(0)}(x)\left(1-\hbar d_{\epsilon_{a}}^{\dagger}(x)+o(\hbar)\right),
$$

where

$$
d_{\epsilon_{a}}^{(0)}(x):=\left(1-x_{a}^{2}\right)^{2} \prod_{p(\neq a)}\left(1-x_{a} / x_{p}\right)\left(1-x_{a} x_{p}\right)
$$

and

$$
\begin{aligned}
d_{\epsilon_{a}}^{\dagger}(x) & :=\alpha \frac{1+x_{a}^{2}}{1-x_{a}^{2}}+2 \beta \frac{x_{a}}{1-x_{a}^{2}} \\
& +c\left(\frac{x_{a}^{2}}{1-x_{a}^{2}}-\sum_{p=1}^{a-1} \frac{x_{a}}{x_{a}-x_{p}}\right)+k \sum_{p(\neq a)}\left(\frac{1}{1-x_{a} x_{p}}-\frac{x_{a}}{x_{a}-x_{p}}\right)
\end{aligned}
$$

To calculate the limit of $\widetilde{S}_{\epsilon_{a}}$ as $\hbar \rightarrow 0$, we use the accessory generators $\widetilde{T}_{i}(1 \leq$ $i \leq n)$ and the relations

$$
\begin{aligned}
& s_{i} \widetilde{T}_{i}+\widetilde{T}_{i} s_{i}=k s_{i} \quad(1 \leq i<n), \quad s_{n} \widetilde{T}_{n}+\widetilde{T}_{n} s_{n}=k_{n} s_{n}, \\
& \widetilde{T}_{i} x_{i}=x_{i+1}\left(\widetilde{T}_{i}-k\right), \quad x_{i} \widetilde{T}_{i}=\left(\widetilde{T}_{i}-k\right) x_{i+1} \quad(1 \leq i<n), \\
& \widetilde{T}_{n} x_{n}=x_{n}^{-1}\left(\widetilde{T}_{i}-k_{n}\right)-k_{n}^{*}
\end{aligned}
$$

derived from the relations (i) and (iii) in Definition 4.2, Then we obtain

$$
\widetilde{S}_{\epsilon_{a}}=d_{\epsilon_{a}}^{(0)}(x)\left(1+\hbar \widetilde{S}_{\epsilon_{a}}^{\dagger}+o(\hbar)\right),
$$

where

$$
\begin{aligned}
\widetilde{S}_{\epsilon_{a}}^{\dagger} & :=\Phi_{a}-c\left(\frac{x_{a}^{2}}{1-x_{a}^{2}}-\sum_{p=1}^{a-1} \frac{x_{a}}{x_{a}-x_{p}}\right) \\
\Phi_{a} & :=y_{a}+2 \frac{\alpha+\beta x_{a}}{x_{a}^{2}-1} r_{a} \\
& +k\left(\sum_{p=1}^{a-1} \frac{x_{a}}{x_{a}-x_{p}} s_{p a}+\sum_{p=a+1}^{n} \frac{x_{p}}{x_{a}-x_{p}} s_{a p}+\sum_{p(\neq a)} \frac{1}{x_{a} x_{p}-1} \tilde{s}_{a p}\right)
\end{aligned}
$$


Note that

where

$$
d_{\epsilon_{a}}^{\dagger}(x)+\widetilde{S}_{\epsilon_{a}}^{\dagger}=\Phi_{a}+c x_{a} \frac{\partial \log U(x)}{\partial x_{a}}
$$

$$
\begin{aligned}
U(x) & :=\prod_{p=1}^{n}\left(x_{p}^{\frac{k(n-1)+\alpha}{c}}\left(1+x_{p}\right)^{\frac{\beta-\alpha}{c}}\left(1-x_{p}\right)^{-\frac{\beta+\alpha}{c}}\right) \\
& \times \prod_{1 \leq p<l \leq n}\left(\left(1-x_{p} x_{l}\right)\left(x_{p}-x_{l}\right)\right)^{-\frac{k}{c}} .
\end{aligned}
$$

Suppose that $F=F(X \mid Y) \in H_{0}^{\mathbb{K}}$ is expanded as in (4.13). Then we have

$$
\left(\epsilon_{a}, 1\right) F-F=\hbar^{M+1}\left(c x_{a} \frac{\partial \bar{G}}{\partial x_{a}}+o(1)\right) .
$$

Hence the trigonometric degeneration of (4.17) is given by

$$
\left(c x_{a} \frac{\partial}{\partial x_{a}}+L_{a}+c x_{a} \frac{\partial \log U(x)}{\partial x_{a}}\right) \bar{G}=0,
$$

where

$$
\begin{aligned}
L_{a} & :=\eta_{L}\left(y_{a}\right)+2 \frac{\alpha+\beta x_{a}}{x_{a}^{2}-1} \eta_{L}\left(r_{a}\right) \\
& +k\left(\sum_{p=1}^{a-1} \frac{x_{a}}{x_{a}-x_{p}} \eta_{L}\left(s_{p a}\right)+\sum_{p=a+1}^{n} \frac{x_{p}}{x_{a}-x_{p}} \eta_{L}\left(s_{a p}\right)+\sum_{p(\neq a)} \frac{1}{x_{a} x_{p}-1} \eta_{L}\left(\tilde{s}_{a p}\right)\right) .
\end{aligned}
$$

4.6. Embedding into the compatible system. As was seen in subsections 4.4 and 4.5, the trigonometric degeneration of the bispectral qKZ equation is the system of equations (4.16) and (4.19) for $\bar{G} \in \mathbb{C}\left[W_{0}\right]^{\overline{\mathbb{K}}}$. Now define $G \in \mathbb{C}\left[W_{0}\right]^{\overline{\mathbb{K}}}$ by

$$
G(x \mid y)=U(x) \bar{G}(x \mid y),
$$

where $U(x)$ is given by (4.18). Note that the operator $\Delta_{m} \Psi_{m}$ commutes with multiplication by any function in $x$. Hence the modified function $G$ also satisfies (4.16). Thus we obtain the system of equations

$$
\begin{array}{cc}
\Delta_{m} \Psi_{m}(G)=-p_{m}^{(0)}(y) G & (1 \leq m \leq n), \\
\left(c x_{a} \frac{\partial}{\partial x_{a}}+L_{a}\right) G=0 & (1 \leq a \leq n)
\end{array}
$$

for $G \in \mathbb{C}\left[W_{0}\right]^{\overline{\mathbb{K}}}$. In this subsection we prove that the above system of equations is contained in our compatible system $(3.2)$. To this end we realize the $(\bar{H}, \mathbb{C}[W])$ bimodule $\mathbb{C}\left[W_{0}\right]^{\overline{\mathbb{K}}}$ in the scalar extension $\left(V^{\otimes n}\right)^{\overline{\mathbb{K}}}:=\overline{\mathbb{K}} \otimes_{\mathbb{C}} V^{\otimes n}$ as follows.

First we define the left action of $W_{0}$ on $V$ by

$$
s_{i}\left(v_{a}\right)=v_{\sigma_{i}(a)}, \quad s_{i}\left(v_{\bar{a}}\right)=v_{\overline{\sigma_{i}(a)}} \quad(1 \leq i<n),
$$


where $\sigma_{i}:=(i, i+1)$ is the transposition, and

$$
s_{n}\left(v_{a}\right)=\left\{\begin{array}{ll}
v_{\bar{n}} & (a=n) \\
v_{a} & (a \neq n),
\end{array} \quad s_{n}\left(v_{\bar{a}}\right)= \begin{cases}v_{n} & (a=n) \\
v_{\bar{a}} & (a \neq n) .\end{cases}\right.
$$

View $V^{\otimes n}$ as a tensor representation of $W_{0}$. We extend it to the $\overline{\mathbb{K}}$-linear action on $\left(V^{\otimes n}\right)^{\overline{\mathbb{K}}}$. Let $\left(V^{\otimes n}\right)_{0}:=\mathbb{C}\left[W_{0}\right]\left(v_{1} \otimes \cdots \otimes v_{n}\right)$ be the cyclic submodule and $\left(V^{\otimes n}\right)_{0}^{\overline{\mathbb{K}}}:=$ $\overline{\mathbb{K}} \otimes_{\mathbb{C}}\left(V^{\otimes n}\right)_{0}$. Denote the left action on $\left(V^{\otimes n}\right)_{0}^{\bar{K}}$ by $\rho_{L}: \mathbb{C}\left[W_{0}\right] \rightarrow \operatorname{End}_{\overline{\mathbb{K}}}\left(V^{\otimes n}\right)_{0}^{\bar{K}}$.

Note that the operators $A_{a}(y), B_{a}(x)$ and $Q_{m}(x \mid y)$ are $\overline{\mathbb{K}}$-linear, and hence belong to $\operatorname{End}_{\overline{\mathbb{K}}}\left(V^{\otimes n}\right)_{0}^{\bar{K}}$.

Lemma 4.3. The following relations hold on $\left(V^{\otimes n}\right)_{0}^{\overline{\mathbb{K}}}$ :

$$
\begin{aligned}
& A_{i}(y) \rho_{L}\left(s_{i}\right)=\rho_{L}\left(s_{i}\right) A_{i+1}(y)+k \quad(1 \leq i<n), \\
& A_{n}(y) \rho_{L}\left(s_{n}\right)=-\rho_{L}\left(s_{n}\right) A_{n}(y)+2 \alpha, \\
& {\left[A_{i}(y), \rho_{L}\left(s_{j}\right)\right]=0 \quad(|i-j|>1 \text { or }(i, j)=(n-1, n)) .}
\end{aligned}
$$

From Lemma 4.3 the action $\rho_{L}$ is extended to that of $\bar{H}$ which maps $y_{a} \mapsto A_{a}(y)$. We also denote it by $\rho_{L}$. Now consider the $\overline{\mathbb{K}}$-linear map

$$
\phi: \mathbb{C}\left[W_{0}\right]^{\bar{K}} \rightarrow\left(V^{\otimes n}\right)_{0}^{\bar{K}}, \quad w \mapsto \rho_{L}(w)\left(v_{1} \otimes \cdots \otimes v_{n}\right) \quad\left(w \in W_{0}\right) .
$$

Proposition 4.4. The map $\phi$ gives an isomorphism between the left $\bar{H}$-modules $\left(\eta_{L}, \mathbb{C}\left[W_{0}\right]^{\overline{\mathbb{K}}}\right)$ and $\left(\rho_{L},\left(V^{\otimes n}\right)_{0}^{\bar{K}}\right)$. In particular we have $\phi \eta_{L}\left(y_{a}\right) \phi^{-1}=A_{a}(y)$.

Proof. It is clear that $\phi$ commutes with the left action of $\mathbb{C}\left[W_{0}\right]$. From $A_{a}(y)\left(v_{1} \otimes\right.$ $\left.\cdots \otimes v_{n}\right)=y_{a} v_{1} \otimes \cdots \otimes v_{n}$ and Lemma 4.3, $\phi$ commutes also with the action of $y_{a} \in \bar{H}(1 \leq a \leq n)$.

Recall that $\mathbb{C}\left[W_{0}\right]^{\overline{\mathbb{K}}}$ is a right $\mathbb{C}[W]$-module with the action $\eta_{R}$ (4.10). Now we define a right action of $\mathbb{C}[W]$ on $\left(V^{\otimes n}\right)_{0}^{\overline{\mathbb{K}}}$ :

Lemma 4.5. There exists an anti-algebra homomorphism $\rho_{R}: \mathbb{C}[W] \rightarrow \operatorname{End}_{\overline{\mathbb{K}}}\left(V^{\otimes n}\right)_{0}^{\mathbb{K}}$ such that

$$
\rho_{R}\left(s_{0}\right)=T_{1}(x), \quad \rho_{R}\left(s_{i}\right)=P_{i, i+1}(1 \leq i<n), \quad \rho_{R}\left(s_{n}\right)=T_{n}(\underline{1}) .
$$

Lemma 4.5 follows from $T(x)^{2}=1$ and the braid relation $P_{i, i+1} P_{i+1, i+2} P_{i, i+1}=$ $P_{i+1, i+2} P_{i, i+1} P_{i+1, i+2}(1 \leq i \leq n-2)$. We denote the right action of $\mathbb{C}[W]$ on $\left(V^{\otimes n}\right)_{0}^{\overline{\mathbb{K}}}$ by $\rho_{R}$.

Proposition 4.6. The map $\phi$ commutes with the right action of $\mathbb{C}[W]$ on $\mathbb{C}\left[W_{0}\right]^{\overline{\mathbb{K}}}$ and $\left(V^{\otimes n}\right)_{0}^{\bar{K}}$. Therefore $\phi$ is an isomorphism between $(\bar{H}, \mathbb{C}[W])$-bimodules.

Proof. Set $v^{\dagger}:=v_{1} \otimes \cdots \otimes v_{n}$. For $w \in W_{0}$ we have

$$
\begin{aligned}
& \phi\left(w s_{i}\right)=w s_{i} v^{\dagger}=w P_{i, i+1} v^{\dagger}=P_{i, i+1} w v^{\dagger}=P_{i, i+1} \phi(w) \quad(1 \leq i<n), \\
& \phi\left(w s_{n}\right)=w s_{n} v^{\dagger}=w T_{n}(\underline{1}) v^{\dagger}=T_{n}(\underline{1}) w v^{\dagger}=T_{n}(\underline{1}) \phi(w) .
\end{aligned}
$$


If $w x_{1}^{-1}$ is equal to $x_{j}^{-1} w$ (resp. $\left.x_{j} w\right)$ in $\mathbb{C}[W]$, the first component of $w r_{1} v^{\dagger} \in V^{\otimes n}$ is $v_{\bar{j}}\left(\right.$ resp. $\left.v_{j}\right)$. Hence we get

$$
\phi\left(w s_{0}\right)=\phi\left(w x_{1}^{-1} r_{1}\right)=\phi\left(x_{j}^{-1} w r_{1}\right)=x_{j}^{-1} \phi\left(w r_{1}\right)=T_{1}(x) w v^{\dagger}=T_{1}(x) \phi(w) .
$$

Now we send the equations (4.21) and (4.22) on $\mathbb{C}\left[W_{0}\right]$ by $\phi$. First consider (4.21). Note that the map $\phi$ commutes with $\nu(w)(\forall w \in W)$ since $\phi$ is $\overline{\mathbb{K}}$-linear. From (4.15) and Proposition 4.6 we find

$$
\begin{aligned}
& \phi\left(\Delta_{m} \Psi_{m}\right) \phi^{-1}=-\left(\left(y_{m}-y_{m+1}\right) P_{m, m+1}-k\right) \cdots\left(\left(y_{m}-y_{n}\right) P_{n-1, n}-k\right) \\
& \times\left(y_{m} T_{n}(\underline{1})-\alpha\right)\left(\left(y_{m}+y_{n}\right) P_{n-1, n}-k\right) \cdots\left(\left(y_{m}+y_{m+1}\right) P_{m, m+1}-k\right) \\
& \times\left(\left(y_{m}+y_{m-1}\right) P_{m-1, m}-k\right) \cdots\left(\left(y_{m}+y_{1}\right) P_{1,2}-k\right)\left(\left(y_{m}-c / 2\right) T_{1}(x)-\beta\right) \\
& \times\left(\left(y_{m}-y_{1}-c\right) P_{1,2}-k\right) \cdots\left(\left(y_{m}-y_{m-1}-c\right) P_{m-1, m}-k\right) \Delta_{m} .
\end{aligned}
$$

Using

$$
\lambda P-k=(\lambda-k) P R(\lambda)^{-1}, \quad \lambda T(x)-\beta=(\lambda-\beta) K(\lambda \mid x, \beta)^{-1},
$$

we obtain

$$
\phi\left(\Delta_{m} \Psi_{m}\right) \phi^{-1}=-p_{m}^{(0)}(y) Q_{m}(x \mid y)^{-1} \Delta_{m} .
$$

Hence, sending (4.21) by $\phi$, we get the boundary rational qKZ equation

$$
\Delta_{m} \phi(G)=Q_{m}(x \mid y) \phi(G) \quad(1 \leq m \leq n)
$$

on $\left(V^{\otimes n}\right)_{0}$.

Next let us consider (4.22). Note that the following equalities hold on $\left(V^{\otimes n}\right)_{0}$ :

$$
\begin{aligned}
& \sum_{j=1}^{n} \bar{E}_{a a}^{(j)}=\rho_{L}\left(r_{a}\right), \quad \mathbf{Y}_{a a}+\mathbf{Z}_{a a}=0, \\
& \mathbf{X}_{a b}+\mathbf{X}_{b a}=\rho_{L}\left(s_{a b}\right), \quad \mathbf{Y}_{a b}+\mathbf{Z}_{a b}=\rho_{L}\left(\tilde{s}_{a b}\right) \quad(a \neq b) .
\end{aligned}
$$

Therefore we have

$$
\phi L_{a} \phi^{-1}=A_{a}(y)+B_{a}(x)=L_{a}(x \mid y) .
$$

The Euler operator $x_{a} \frac{\partial}{\partial x_{a}}$ commutes with $\phi$. Consequently, sending (4.22) by $\phi$, we obtain the differential equation

$$
D_{a}(x \mid y) \phi(G)=0 \quad(1 \leq a \leq n) .
$$

As a result we find

Proposition 4.7. The compatible system (3.2) with $N=n$ restricted to $\left(V^{\otimes n}\right)_{0}$ is equivalent to the trigonometric degeneration (4.16) and (4.19) of the bispectral $q K Z$ equation of type $\left(C_{n}^{\vee}, C_{n}\right)$ through the gauge transform (4.20). 


\section{INTEGRAL FORMULA FOR SOLUTIONS IN A SPECIAL CASE}

We construct an integral formula for solutions to the compatible system (3.2) with

$$
\alpha=\beta=k / 2
$$

and the variable $x$ restricted to the hyperplane

$$
x=\left(x_{1}, 1, \ldots, 1\right) .
$$

Hence only one differential operator $D_{1}(x \mid y)$ enters in the system. In the following we set

$$
x_{1}=e^{2 \pi i \lambda}
$$

and assume that

$$
\operatorname{Im} c>0, \quad \operatorname{Im} k>0
$$

for simplicity's sake.

Our solutions take values in a $2 n$-dimensional subspace of $V^{\otimes n}$ determined as follows. Denote by $\tilde{V}$ the subspace of $V$ spanned by $v_{a}$ and $v_{\bar{a}}(2 \leq a \leq N)$. We fix a non-zero vector $\tilde{v} \in \tilde{V}^{\otimes(n-1)}$ satisfying

$$
P_{i, i+1} \tilde{v}=\tilde{v} \quad(1 \leq \forall i \leq n-2), \quad T_{n-1}(\underline{1}) \tilde{v}=\tilde{v} .
$$

Now define the vectors $u_{j} \in V^{\otimes 2 n}(1 \leq j \leq 2 n)$ by

$$
u_{j}:=P_{1, j}\left(v_{1} \otimes \tilde{v}\right), \quad u_{2 n+1-j}:=P_{j, n}\left(\tilde{v} \otimes v_{\overline{1}}\right) \quad(1 \leq j \leq n),
$$

where $P_{1,1}=P_{n, n}=i d$. Our solutions take values in the subspace $\mathcal{V}:=\oplus_{j=1}^{2 n} \mathbb{C} u_{j}$.

Define the rational functions $g_{j}(t)=g_{j}(t \mid y)(1 \leq j \leq 2 n)$ by

$$
\begin{aligned}
g_{j}(t) & :=\frac{1}{t-y_{j}} \prod_{p=1}^{j-1} \frac{t-y_{p}-k}{t-y_{p}}, \\
g_{2 n+1-j}(t) & :=\frac{1}{t+y_{j}} \prod_{p=j+1}^{n} \frac{t+y_{p}-k}{t+y_{p}} \prod_{p=1}^{n} \frac{t-y_{p}-k}{t-y_{p}},
\end{aligned}
$$

for $1 \leq j \leq n$. Set $\mathcal{G}:=\sum_{j=1}^{2 n} \mathbb{C} g_{j}(t)$.

Denote by $\mathcal{W}$ the $\mathbb{C}$-vector space spanned by the functions in the form

$$
\frac{P\left(e^{2 \pi i t / c}\right)}{\prod_{p=1}^{n}\left(1-e^{2 \pi i\left(t-y_{p}\right) / c}\right)\left(1-e^{2 \pi i\left(t+y_{p}\right) / c}\right)},
$$

where $P$ is a polynomial whose coefficients are entire and periodic functions in $y_{1}, \ldots, y_{n}$ with period $c$.

Now we define a pairing $I$ between $\mathcal{G}$ and $\mathcal{W}$ by

$$
I(g, W):=\int_{C(y)} \varphi(t \mid y) g(t) W\left(e^{2 \pi i t / c}\right) d t \quad(g \in \mathcal{G}, W \in \mathcal{W})
$$


where the kernel function $\varphi$ is defined by

$$
\varphi(t \mid y):=e^{-\frac{2 \pi i \lambda}{c} t} \prod_{p=1}^{n} \frac{\Gamma\left(\frac{t-y_{p}-k}{-c}\right) \Gamma\left(\frac{t+y_{p}-k}{-c}\right)}{\Gamma\left(\frac{t-y_{p}}{-c}\right) \Gamma\left(\frac{t+y_{p}}{-c}\right)} .
$$

The contour $C(y)$ is a deformation of the real line $(-\infty,+\infty)$ such that the poles at $\pm y_{p}+k+c \mathbb{Z}_{\geq 0}(1 \leq p \leq n)$ are above $C(y)$ and the poles at $\pm y_{p}+c \mathbb{Z}_{\leq 0}(1 \leq p \leq n)$ are below $C(y)$. Suppose that $W\left(e^{2 \pi i t / c}\right)$ is given by (5.1). From the Stirling formula we see that the integral (5.2) converges if

$$
\operatorname{Re} \lambda<\operatorname{deg} P<\operatorname{Re} \lambda+2 n \text {. }
$$

For $W \in \mathcal{W}$ satisfying the degree condition (5.3) we set

$$
f(\lambda \mid y):=\sum_{j=1}^{2 n} I\left(g_{j}, W\right) u_{j}
$$

Proposition 5.1. The function $f$ satisfies the boundary rational $q K Z$ equation (2.4) with $\alpha=\beta=k / 2$ and $x=\left(e^{2 \pi i \lambda}, 1, \ldots, 1\right)$.

Proof. In the proof below we need to signify the dependence of $W \in \mathcal{W}$ on $y$. For that purpose we set

$$
\begin{aligned}
& \tilde{g}\left(t \mid y^{\prime}\right)=\sum_{j=1}^{2 n} g_{j}\left(t \mid y^{\prime}\right) u_{j} \\
& \tilde{f}\left(\lambda\left|y^{\prime}\right| y\right)=\int_{C\left(y^{\prime}\right)} \varphi\left(t \mid y^{\prime}\right) \tilde{g}\left(t \mid y^{\prime}\right) W\left(e^{2 \pi i t / c} \mid y\right) d t
\end{aligned}
$$

for $y^{\prime}=\left(y_{1}^{\prime}, \ldots, y_{n}^{\prime}\right)$ such that each coordinate $y_{j}^{\prime}$ belongs to the set $\left\{ \pm y_{a}+c l \mid 1 \leq\right.$ $a \leq n, l \in \mathbb{Z}\}$.

By direct calculation we get

$$
\begin{aligned}
& P_{l, l+1} R_{l, l+1}\left(y_{l}-y_{l+1}\right) \tilde{g}\left(\ldots, y_{l}, y_{l+1}, \ldots\right)=\tilde{g}\left(\ldots, y_{l+1}, y_{l}, \ldots\right) \quad(1 \leq l \leq n-1), \\
& K_{n}\left(y_{n} \mid \underline{1}, k / 2\right) \tilde{g}\left(y_{1}, \ldots, y_{n-1}, y_{n}\right)=\tilde{g}\left(y_{1}, \ldots, y_{n-1},-y_{n}\right) .
\end{aligned}
$$

Since $\varphi(t \mid y)$ and $C(y)$ are invariant under the transposition $y_{l} \leftrightarrow y_{l+1}$ and the reflection $y_{n} \rightarrow-y_{n}$, the above equalities where $\tilde{g}(\ldots)$ is replaced by $\tilde{f}(\lambda|\ldots| y)$ also hold. From this fact and the periodicity of $W$ in $y$, it is enough to prove that

$$
K_{1}\left(y_{1}-c / 2 \mid x, k / 2\right) \tilde{f}\left(\lambda\left|-y_{1}, y_{2}, \ldots, y_{n}\right| y\right)=\tilde{f}\left(\lambda\left|y_{1}-c, y_{2}, \ldots, y_{n}\right| y\right)
$$


with $x=\left(e^{2 \pi i \lambda}, 1, \ldots, 1\right)$. We abbreviate $y^{(1)}=\left(-y_{1}, y_{2}, \ldots, y_{n}\right)$ and $y^{(2)}=\left(y_{1}-\right.$ $\left.c, y_{2}, \ldots, y_{n}\right)$. Taking the coefficients of $u_{j}(1 \leq j \leq 2 n)$ in (5.5) we have the equalities to prove:

$$
\begin{aligned}
& \int_{C\left(y^{(1)}\right)} \varphi\left(t \mid y^{(1)}\right) g_{j}\left(t \mid y^{(1)}\right) W\left(e^{2 \pi i t / c}\right) d t \\
& =\int_{C\left(y^{(2)}\right)} \varphi\left(t \mid y^{(2)}\right) g_{j}\left(t \mid y^{(2)}\right) W\left(e^{2 \pi i t / c}\right) d t \quad(j \neq 1,2 n) \\
& \int_{C\left(y^{(1)}\right)} \varphi\left(t \mid y^{(1)}\right) \frac{e^{2 \pi i \lambda}\left(y_{1}-c / 2\right) g_{2 n}\left(t \mid y^{(1)}\right)+(k / 2) g_{1}\left(t \mid y^{(1)}\right)}{y_{1}-c / 2+k / 2} W\left(e^{2 \pi i t / c}\right) d t \\
& =\int_{C\left(y^{(2)}\right)} \varphi\left(t \mid y^{(2)}\right) g_{1}\left(t \mid y^{(2)}\right) W\left(e^{2 \pi i t / c}\right) d t \\
& \int_{C\left(y^{(1)}\right)} \varphi\left(t \mid y^{(1)}\right) \frac{e^{-2 \pi i \lambda}\left(y_{1}-c / 2\right) g_{1}\left(t \mid y^{(1)}\right)+(k / 2) g_{2 n}\left(t \mid y^{(1)}\right)}{y_{1}-c / 2+k / 2} W\left(e^{2 \pi i t / c}\right) d t \\
& =\int_{C\left(y^{(2)}\right)} \varphi\left(t \mid y^{(2)}\right) g_{2 n}\left(t \mid y^{(2)}\right) W\left(e^{2 \pi i t / c}\right) d t .
\end{aligned}
$$

First we prove (5.6). If $j \neq 1,2 n$, the functions $g_{j}\left(t \mid y^{(1)}\right)$ and $g_{j}\left(t \mid y^{(2)}\right)$ have the common factor independent on $y_{1}$ and are in the form

$$
\begin{aligned}
& g_{j}\left(t \mid y^{(1)}\right)=\frac{t+y_{1}-k}{t+y_{1}} w_{j}\left(t \mid y_{2}, \ldots, y_{n}\right), \\
& g_{j}\left(t \mid y^{(2)}\right)=\frac{t-y_{1}-k+c}{t-y_{1}+c} w_{j}\left(t \mid y_{2}, \ldots, y_{n}\right) .
\end{aligned}
$$

Note that $\varphi\left(t \mid y^{(1)}\right)=\varphi(t \mid y)$. Moreover, from

$$
\varphi\left(t \mid y^{(2)}\right)=\varphi(t \mid y) \frac{t-y_{1}+c}{t-y_{1}-k+c} \frac{t+y_{1}-k}{t+y_{1}}
$$

we have

$$
\varphi\left(t \mid y^{(2)}\right) g_{j}\left(t \mid y^{(2)}\right)=\varphi(t \mid y) \frac{t+y_{1}-k}{t+y_{1}} w_{j}\left(t \mid y_{2}, \ldots, y_{n}\right)=\varphi\left(t \mid y^{(1)}\right) g_{j}\left(t \mid y^{(1)}\right) .
$$

Thus the integrands in the both hand sides of (5.6) are the same. Since the integrand has no poles at $-y_{1}+k,-y_{1}+c, y_{1}$ and $y_{1}+k-c$, we can deform $C\left(y^{(1)}\right)$ to $C\left(y^{(2)}\right)$ without crossing poles. Thus we obtain (5.6).

In the rest we only prove (5.7). The proof of (5.8) is similar. We separate the integrand in the left hand side and first consider

$$
\frac{y_{1}-c / 2}{y_{1}-c / 2+k / 2} \int_{C\left(y^{(1)}\right)} \varphi\left(t \mid y^{(1)}\right) e^{2 \pi i \lambda} g_{2 n}\left(t \mid y^{(1)}\right) W\left(e^{2 \pi i t / c}\right) d t
$$


We have

$$
\varphi\left(t \mid y^{(1)}\right) e^{2 \pi i \lambda} g_{2 n}\left(t \mid y^{(1)}\right)=\varphi(t-c \mid y) \frac{1}{t-y_{1}-k} .
$$

Changing $t \rightarrow t+c$ we see that the integral (5.10) is equal to

$$
\frac{y_{1}-c / 2}{y_{1}-c / 2+k / 2} \int_{C\left(y^{(1)}\right)-c} \varphi(t \mid y) \frac{1}{t+c-y_{1}-k} W\left(e^{2 \pi i t / c}\right) d t .
$$

Since the integrand has no poles at $\pm y_{j}(1 \leq j \leq n)$, the contour $C\left(y^{(1)}\right)-c$ can be deformed to the contour $C^{\prime}$, which is a deformation of the real line such that the points $-y_{1}+k+c \mathbb{Z}_{\geq 0}, y_{1}+k+c \mathbb{Z}_{\geq-1}$ and $\pm y_{j}+k+c \mathbb{Z}_{\geq 0}(2 \leq j \leq n)$ are above $C^{\prime}$, and the points $-y_{1}+c \mathbb{Z}_{\leq 0}, y_{1}+c \mathbb{Z}_{\leq-1}$ and $\pm y_{j}+c \mathbb{Z}_{\leq 0}(2 \leq j \leq n)$ are below $C^{\prime}$. The integrand of the rest part of the left hand side of (5.7) has no pole at $y_{1}$, hence the contour $C\left(y^{(1)}\right)$ can be deformed to $C^{\prime}$. As a result the left hand side becomes

$$
\begin{aligned}
& \int_{C^{\prime}} \varphi(t \mid y)\left(\frac{y_{1}-c / 2}{y_{1}-c / 2+k / 2} \frac{1}{t+c-y_{1}-k}+\frac{k / 2}{y_{1}-c / 2+k / 2} \frac{1}{t+y_{1}}\right) W\left(e^{2 \pi i t / c}\right) d t \\
& =\int_{C^{\prime}} \varphi(t \mid y) \frac{1}{t+c-y_{1}-k} \frac{t+y_{1}-k}{t+y_{1}} W\left(e^{2 \pi i t / c}\right) d t .
\end{aligned}
$$

On the other hand, using (5.9) we see that the right hand side of (5.7) is equal to

$$
\int_{C\left(y^{(2)}\right)} \varphi(t \mid y) \frac{1}{t+c-y_{1}-k} \frac{t+y_{1}-k}{t+y_{1}} W\left(e^{2 \pi i t / c}\right) d t .
$$

Since the integrand is regular at $t=-y_{1}+c$, the contour $C\left(y^{(2)}\right)$ can be deformed to $C^{\prime}$ without crossing poles. Thus we obtain the equality (5.7).

Proposition 5.2. If $\alpha=\beta=k / 2$ and $x=\left(e^{2 \pi i \lambda}, 1, \ldots, 1\right)$, we have

$$
D_{1}(x \mid y) f(\lambda \mid y)=-\frac{k e^{2 \pi i \lambda}}{e^{2 \pi i \lambda}-1} f(\lambda \mid y) .
$$

Proof. Since $\mathbf{Y}_{11}$ and $\mathbf{Z}_{11}$ act as zero on $\mathcal{V}$, we find

$$
\begin{aligned}
\left.D_{1}(x \mid y)\right|_{\mathcal{V}}=\frac{c}{2 \pi i} \frac{\partial}{\partial \lambda}+\sum_{j=1}^{n} y_{j}\left(e_{11}^{(j)}-e_{\overline{1} \overline{1}}^{(j)}\right) & +\frac{k}{e^{2 \pi i \lambda}-1}\left(\sum_{j=1}^{n} e_{\overline{1} 1}^{(j)}+\sum_{p=2}^{N}\left(\mathbf{X}_{p 1}+\mathbf{Z}_{1 p}\right)\right) \\
& +\frac{k e^{2 \pi i \lambda}}{e^{2 \pi i \lambda}-1}\left(\sum_{j=1}^{n} e_{1 \overline{1}}^{(j)}+\sum_{p=2}^{N}\left(\mathbf{X}_{1 p}+\mathbf{Y}_{1 p}\right)\right)
\end{aligned}
$$

if $x=\left(e^{2 \pi i \lambda}, 1, \ldots, 1\right)$ and $\alpha=\beta=k / 2$. For $1 \leq j \leq n$ we have

$$
\sum_{p=2}^{N}\left(\mathbf{X}_{p 1}+\mathbf{Z}_{1 p}\right) v_{j}=\sum_{l=j+1}^{2 n-j} v_{l}+\sum_{l=2 n+2-j}^{2 n} v_{l}, \quad \sum_{p=2}^{N}\left(\mathbf{X}_{1 p}+\mathbf{Y}_{1 p}\right) v_{j}=\sum_{l=1}^{j-1} v_{l}
$$


and

$$
\sum_{p=2}^{N}\left(\mathbf{X}_{p 1}+\mathbf{Z}_{1 p}\right) v_{2 n+1-j}=\sum_{l=2 n+2-j}^{2 n} v_{l}, \quad \sum_{p=2}^{N}\left(\mathbf{X}_{1 p}+\mathbf{Y}_{1 p}\right) v_{2 n+1-j}=\sum_{l=1}^{j-1} v_{l}+\sum_{l=j+1}^{2 n-j} v_{l} .
$$

From the calculation above we obtain

$$
D_{1}(x \mid y) f(\lambda \mid y)=\sum_{j=1}^{2 n} I\left(h_{j}, W\right) u_{j}
$$

where the rational functions $h_{j}(1 \leq j \leq 2 n)$ are given by

$$
\begin{aligned}
& h_{j}(t \mid y)=-\left(t-y_{j}\right) g_{j}(t \mid y)+\frac{k}{e^{2 \pi i \lambda}-1} \sum_{l=1}^{j-1} g_{l}(t \mid y)+\frac{k e^{2 \pi i \lambda}}{e^{2 \pi i \lambda}-1} \sum_{l=j+1}^{2 n} g_{l}(t \mid y), \\
& h_{2 n+1-j}(t \mid y)=-\left(t+y_{j}\right) g_{j}(t \mid y)+\frac{k}{e^{2 \pi i \lambda}-1} \sum_{l=1}^{2 n-j} g_{l}(t \mid y)+\frac{k e^{2 \pi i \lambda}}{e^{2 \pi i \lambda}-1} \sum_{l=2 n+2-j}^{2 n} g_{l}(t \mid y)
\end{aligned}
$$

for $1 \leq j \leq n$.

Note that the functions $g_{j}(1 \leq j \leq 2 n)$ are rewritten as

$$
\begin{aligned}
g_{j}(t \mid y) & =\frac{1}{k}\left(\prod_{l=1}^{j-1} \frac{t-y_{l}-k}{t-y_{l}}-\prod_{l=1}^{j} \frac{t-y_{l}-k}{t-y_{l}}\right) \\
g_{2 n+1-j}(t \mid y) & =\frac{1}{k}\left(\prod_{l=j+1}^{n} \frac{t+y_{l}-k}{t+y_{l}}-\prod_{l=j}^{n} \frac{t+y_{l}-k}{t+y_{l}}\right) \prod_{l=1}^{n} \frac{t-y_{l}-k}{t-y_{l}}
\end{aligned}
$$

for $1 \leq j \leq n$. Using these formulas we get

$$
h_{j}(t \mid y)=-\frac{k e^{2 \pi i \lambda}}{e^{2 \pi i \lambda}-1} g_{j}(t \mid y)+\frac{1}{1-e^{2 \pi i \lambda}}\left(1-e^{2 \pi i \lambda} \prod_{l=1}^{n} \frac{t-y_{l}-k}{t-y_{l}} \frac{t+y_{l}-k}{t+y_{l}}\right)
$$

for $1 \leq j \leq 2 n$. The last term in the right hand side is related to the kernel function $\varphi$ by

$$
e^{2 \pi i \lambda} \prod_{l=1}^{n} \frac{t-y_{l}-k}{t-y_{l}} \frac{t+y_{l}-k}{t+y_{l}}=\frac{\varphi(t-c \mid y)}{\varphi(t \mid y)} .
$$

Therefore we have

$$
\begin{aligned}
& \int_{C(y)} \varphi(t \mid y)\left(1-e^{2 \pi i \lambda} \prod_{l=1}^{n} \frac{t-y_{l}-k}{t-y_{l}} \frac{t+y_{l}-k}{t+y_{l}}\right) W\left(e^{2 \pi i t / c}\right) d t \\
& =\int_{C(y)}(\varphi(t \mid y)-\varphi(t-c \mid y)) W\left(e^{2 \pi i t / c}\right) d t \\
& =\left(\int_{C(y)}-\int_{C(y)-c}\right) \varphi(t \mid y) W\left(e^{2 \pi i t / c}\right) d t .
\end{aligned}
$$


Since the integrand $\varphi(t \mid y) W\left(e^{2 \pi i t / c}\right)$ is regular at $t= \pm y_{j}(1 \leq j \leq n)$, the integral (5.11) is equal to zero. Therefore we obtain

$$
\begin{aligned}
D_{1}(x \mid y) f\left(e^{2 \pi i \lambda} \mid y\right) & =\sum_{j=1}^{2 n} I\left(h_{j}, W\right) u_{j} \\
& =-\frac{k e^{2 \pi i \lambda}}{e^{2 \pi i \lambda}-1} \sum_{j=1}^{2 n} I\left(g_{j}, W\right) u_{j}=-\frac{k e^{2 \pi i \lambda}}{e^{2 \pi i \lambda}-1} f(\lambda \mid y) .
\end{aligned}
$$

The linear operator $L_{1}(x \mid y)$ commutes with multiplication by any function in $x$. Hence, from Proposition 5.1] and Proposition [5.2, we finally get

Theorem 5.3. Set

$$
\tilde{f}(\lambda \mid y):=\left(e^{2 \pi i \lambda}-1\right)^{k / c} f(\lambda \mid y)
$$

where $f$ is defined by (5.4). Then the function $\tilde{f}$ is a solution to the compatible system (3.2) with $\alpha=\beta=k / 2$ and $x=\left(e^{2 \pi i \lambda}, 1, \ldots, 1\right)$ for any $W \in \mathcal{W}$ satisfying (5.3).

Acknowledgements. The research of the author is supported by Grant-in-Aid for Young Scientists (B) No. 20740088. The author is deeply grateful to Saburo Kakei, Masahiro Kasatani, Michitomo Nishizawa and Yoshihisa Saito for valuable discussions.

\section{REFERENCES}

[1] Cantini L., qKZ equations and ground state of the $\mathrm{O}(1)$ loop model with open boundary conditions, arXiv:0903.5050.

[2] Cherednik, I., Quantum Knizhnik-Zamolodchikov equations and affine root systems, Comm. Math. Phys. 150 (1992), no. 1, 109-136.

[3] Cherednik, I., Factorizing particles on a half line, and root systems, Theoret. and Math. Phys. 61 (1984), no. 1, 977-983.

[4] Cherednik, I., Double affine Hecke algebras, London Mathematical Society Lecture Note Series, 319, Cambridge University Press, Cambridge, 2005.

[5] Di Francesco, P. and Zinn-Justin, P., Quantum Knizhnik-Zamolodchikov equation: reflecting boundary conditions and combinatorics, J. Stat. Mech. Theory Exp. 2007, no. 12, P12009.

[6] Etingof, P. and Varchenko, A., Dynamical Weyl groups and applications, Adv. Math. 167 (2002), no. 1, 74-127.

[7] Felder, G., Markov, Y., Tarasov, V. and Varchenko, A., Differential equations compatible with KZ equations, Math. Phys. Anal. Geom. 3 (2000), no. 2, 139-177.

[8] Frenkel, I. B. and Reshetikhin, N. Yu., Quantum affine algebras and holonomic difference equations, Comm. Math. Phys. 146 (1992), no. 1, 1-60.

[9] de Gier, J., Ponsaing A. and Shigechi K., Exact finite size groundstate of the $\mathrm{O}(n=1)$ loop model with open boundaries, J. Stat. Mech. Theory Exp. 2009, no. 4, P04010.

[10] Jimbo, M., Kedem, R., Konno, H., Miwa, T. and Weston, R., Difference equations in spin chains with a boundary, Nuclear Phys. B 448 (1995), no. 3, 429-456. 
[11] van Meer, M. and Stokman, J., Double affine Hecke algebras and bispectral quantum KnizhnikZamolodchikov equations, arXiv:0812.1005.

[12] Mimachi, K., A solution of the quantum Knizhnik Zamolodchikov equation of type $C_{n}$, Comm. Math. Phys. 197 (1998), no. 1, 229-246.

[13] Noumi, M., Macdonald-Koornwinder polynomials and affine Hecke rings, Sūrikaisekikenkyūsho kōkyūroku No. 919 (1995), 44-55 (Japanese).

[14] Sahi, S., Nonsymmetric Koornwinder polynomials and duality, Ann. of Math. (2) 150 (1999), no. $1,267-282$.

[15] Tarasov, V. and Varchenko, A., Difference equations compatible with trigonometric KZ differential equations, Internat. Math. Res. Notices 2000, no. 15, 801-829.

[16] Tarasov, V. and Varchenko, A., Dynamical differential equations compatible with rational $q K Z$ equations, Lett. Math. Phys. 71 (2005), no. 2, 101-108.

Department of Mathematics, Graduate School of Pure and Applied Sciences, Tsukuba University, TSUkuba, IBARAKi 305-8571, JAPAN

E-mail address: takeyama@math.tsukuba.ac.jp 